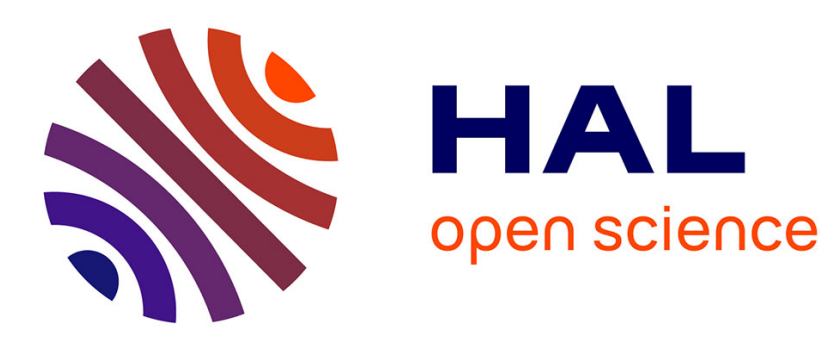

\title{
Wave-Vortex mode coupling in astrophysical accretion disks under combined radial and vertical stratification
}

\author{
Abdelaziz Salhi, T. Lehner, Fabien S. Godeferd, Claude Cambon
}

\section{To cite this version:}

Abdelaziz Salhi, T. Lehner, Fabien S. Godeferd, Claude Cambon. Wave-Vortex mode coupling in astrophysical accretion disks under combined radial and vertical stratification. The Astrophysical Journal, 2013, 771, pp.103. 10.1088/0004-637X/771/2/103 . hal-00931495

\section{HAL Id: hal-00931495 \\ https://hal.science/hal-00931495}

Submitted on 11 Apr 2016

HAL is a multi-disciplinary open access archive for the deposit and dissemination of scientific research documents, whether they are published or not. The documents may come from teaching and research institutions in France or abroad, or from public or private research centers.
L'archive ouverte pluridisciplinaire HAL, est destinée au dépôt et à la diffusion de documents scientifiques de niveau recherche, publiés ou non, émanant des établissements d'enseignement et de recherche français ou étrangers, des laboratoires publics ou privés. 


\title{
WAVE-VORTEX MODE COUPLING IN ASTROPHYSICAL ACCRETION DISKS UNDER COMBINED RADIAL AND VERTICAL STRATIFICATION
}

\author{
A. SAlHi ${ }^{1,2}$, T. LeHNER ${ }^{3}$, F. GODEFERD ${ }^{2}$, AND C. CAMBON ${ }^{2}$ \\ ${ }^{1}$ Département de Physique, Faculté des Sciences de Tunis, 1060 Tunis, Tunisia \\ ${ }^{2}$ Laboratoire de Mécanique des Fluides et d'Acoustique, Ecole Centrale de Lyon, Université de Lyon, UMR 5509, \\ CNRS, INSA, UCB, F-69134 Ecully Cedex, France \\ ${ }^{3}$ LUTH, UMR 8102 CNRS, Observatoire de Paris-Meudon, 5 place de Janssen, F-92195 Meudon, France \\ Received 2011 May 27; accepted 2013 May 8; published 2013 June 21
}

\begin{abstract}
We examine accretion disk flow under combined radial and vertical stratification utilizing a local Cartesian (or "shearing box") approximation. We investigate both axisymmetric and nonaxisymmetric disturbances with the Boussinesq approximation. Under axisymmetric disturbances, a new dispersion relation is derived. It reduces to the Solberg-Hoiland criterion in the case without vertical stratification. It shows that, asymptotically, stable radial and vertical stratification cannot induce any linear instability; Keplerian flow is accordingly stable. Previous investigations strongly suggest that the so-called bypass concept of turbulence (i.e., that fine-tuned disturbances of any inviscid smooth shear flow can reach arbitrarily large transient growth) can also be applied to Keplerian disks. We present an analysis of this process for three-dimensional plane-wave disturbances comoving with the shear flow of a general rotating shear flow under combined stable radial and vertical rotation. We demonstrate that large transient growth occurs for $K_{2} / k_{1} \gg 1$ and $k_{3}=0$ or $k_{1} \sim k_{3}$, where $k_{1}, K_{2}$, and $k_{3}$ are the azimuthal, radial, and vertical components of the initial wave vector, respectively. By using a generalized "wave-vortex" decomposition of the disturbance, we show that the large transient energy growth in a Keplerian disk is mainly generated by the transient dynamics of the vortex mode. The analysis of the power spectrum of total (kinetic+potential) energy in the azimuthal or vertical directions shows that the contribution coming from the vortex mode is dominant at large scales, while the contribution coming from the wave mode is important at small scales. These findings may be confirmed by appropriate numerical simulations in the high Reynolds number regime.
\end{abstract}

Key words: accretion, accretion disks - hydrodynamics - instabilities - turbulence

\section{INTRODUCTION}

Accretion phenomena play an important role in the evolution of many astrophysical systems. However, these phenomena are far from being completely understood. For example, in accretion disks, the collapse of the matter toward the central object is a priori inhibited by the conservation of angular momentum. One usually needs to appeal to turbulence to induce the necessary enhanced transport coefficients responsible for the inflow of matter and the outward transport of angular momentum (through a "turbulent" viscosity coefficient; see Shakura \& Sunyaev 1973). The alpha disk model is a popular example. Several kinds of instabilities can occur in disks. These instabilities are usually considered separately, after having been split into different classes: the magneto-rotational instability (hereafter MRI) for ionized disks, thermal convection effects including different kinds of baroclinic instabilities, and transient growth mechanisms leading to a possible bypass transition to turbulence for purely hydrodynamical disks. Each class is discussed in a number of papers; we quote here only a few of the papers directly related to our present study. The MRI is thought to power magneto-hydrodynamic turbulence, but its driving mechanism still remains unclear. Its dynamics can redistribute angular momentum (see Balbus \& Hawley 1998) in a way that ensures accretion. Our current paper shares important technical material with our recent paper, Salhi et al. (2012). In this recent paper, we developed methods for studying possible transient growth in the case of magnetized disks. For instance, in self-magnetized disks, we studied the dynamo effect with an induced toroidal magnetic field. In this situation, MRI for the vertical magnetic field cannot operate. In contrast, in non-magnetized portions of disks, for example: disks around young low-mass stars, in cataclysmic-variable disks in quiescence, in X-ray transients in quiescence (Stone et al. 2000; Gammie \& Menou 1998; Menou 2000; Johnson \& Gammie 2005a, 2005b), or in purely azimuthal magnetic fields, the MRI cannot operate (see, e.g., Balbus \& Hawley 1998). It is widely believed that these disks are turbulent even though the physical origin of this hydrodynamical turbulence is still highly debated (see, e.g., Yecko 2004; Ji et al. 2006; Balbus 2011). Thermal convection effects could also be important for angular momentum transport. In the presence of stratification, thermal convection effects could lead to baroclinic effects. For example, Klar (2004) studied transient convective baroclinic instabilities due to an entropy gradient; these instabilities produced possible thermal winds. Thermal convection in the presence of differential rotation, together with radial stratification that rendered the disk sub-Keplerian, was also considered by Klar \& Bodenheimer (2003) with an assumed radial negative entropy gradient for proto-planetary disks. A thermal wind was produced in this case.

In this paper, we mainly concentrate on the third topic about transient growth processes in connection with the analogy (within its limitations) between accretion disks and Couette (Couette-Taylor) shear flows (plane and cylindrical ones). In cylindrical flows with a Keplerian profile, the shearing sheet approximation (hereafter SSA; see Goldreich \& Lynden-Bell 1965; Balbus \& Hawley 1998, 2006; Umurhan \& Regev 2004; Regev \& Umurhan 2008; see also Figure 1) is used in many investigations of circumstellar Keplerian. This approximation is also used in the rotating plane of Couette flows that may be of direct interest to astrophysics. However, the validity of the SSA is limited to a local stability analysis only. Also from different recent experiments (see, for example, Paoletti et al. 2012 and references therein), there is still a controversy (see also Balbus 2011) whether a cylindrical Couette-Taylor flow has sufficiently relevant features to mimic correctly in the laboratory the angular momentum transport that may occur in accretion disks. 


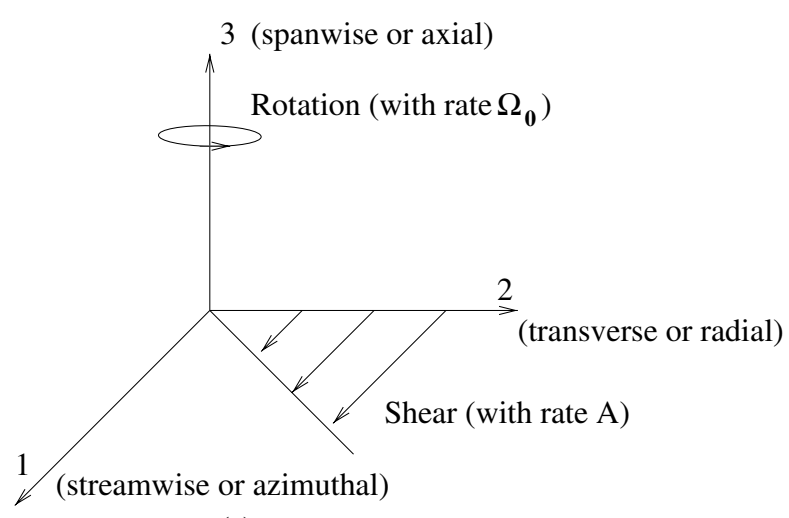

(a)

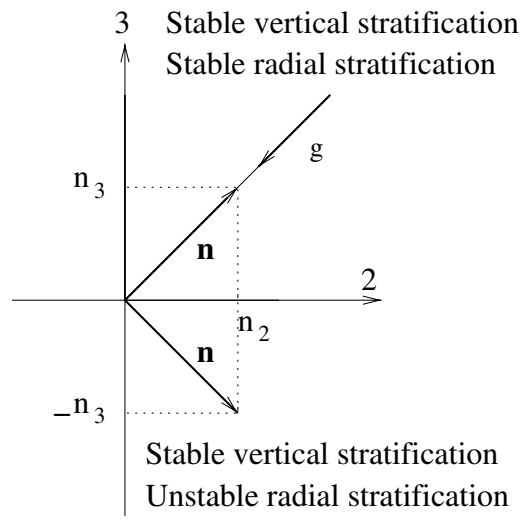

(b)

Figure 1. Illustration of a shearing sheet flow under combined vertical and radial stratification.

We refer the reader a recent study by Schartman et al. (2012) where no net such transport was observed (consistent with the results of Paoletti et al). In these two experiments, there was, however, no density stratification.

The flows under consideration are smooth shear flows (i.e., they present no inflection point in the equilibrium velocity profile). According to theoretical analysis, these flows are linearly stable for all Reynolds numbers up to infinity (see, e.g., Mukhopadhyay et al. 2005). Linear stability analysis, based on eigenspectra (see, e.g., Drazin \& Reid 1981), yields no guarantee that these flows will avoid becoming turbulent. On the other hand, it has been shown that linearly-stable sheared flows can exhibit significant "transient growth" in energy given suitable initial perturbations (see, e.g., Schmid \& Henningson 2001). This last mechanism is called a "bypass transition" to turbulence and provides a possible solution to the problem of explaining the occurrence of turbulence in otherwise spectrally stable shear flows. Transient growth theory has been addressed by a number of authors in the hydrodynamics community (see, e.g., Boberg \& Brosa 1988; Butler \& Farrell 1992; Reddy \& Henningson 1993; Grossmann 2000). It has been shown previously that transient growth results from the non-normal character of the operators describing the linear dynamics in smooth sheared flows. This result arises because the corresponding eigenfunctions are non-orthogonal and can strongly interfere for a finite time, limited by modes phase detuning. This idea has been applied to astrophysical accretion disks to analyze the disks' hydrodynamic turbulence problem. Ioannou \& Kakouris (2001) examined two-dimensional (2D) transient growth in a global disk model (see also Longaretti 2002; Lesur \& Longaretti 2007). Chagelishvili et al. (2003) used the SSA and showed that strong transient growth can occur for a high initial ratio of the azimuthal wavelength to the radial one. In that study, they find a physical explanation for this bypass scenario, as well as a connection between disk flow and examples from fluid dynamics (see also Afshordi et al. 2005). We note that the analytical solution derived by Afshordi et al. (2005) for axisymmetric disturbances (i.e., for an infinite azimuthal wavelength, or equivalently the $m=0$ mode) is the same as the one derived by Salhi \& Cambon (1997). In another study by Salhi et al. (1997), an analytical solution for three-dimensional (3D) vorticity disturbances was derived for the case of a disk with a constant specific angular momentum with $q=2$ for the exponent of the radial dependence of the differential rotation $\Omega(r) \propto r^{-q}$ in the accretion disk ( $q=3 / 2$ for Keplerian orbits). Umurhan \& Regev (2004) performed a nonlinear development of the Chagelishvili et al. (2003) growing mode, while Yecko (2004) and Mukhopadhyay et al. (2005) studied accretion disk (within SSA) under 3D disturbances including both viscosity and rigid boundary conditions (the Reynolds numbers for cold astrophysical disks are believed to be much larger than $10^{6}$, but there is not yet any direct possible measurement of viscosity or a related quantity in disks). These authors found very strong transient growth factors that may trigger hydrodynamic turbulence in disks. In addition, the effect of stratification on Keplerian flows has been addressed by many authors since the combined action of differential rotation and stratification may influence the flow stability and lead to turbulence at a high enough Reynolds number. Dubrulle et al. (2005) discussed the possibility that stable vertically stratified accretion disks are dynamically unstable to nonaxisymmetric disturbances. They found a global instability that is intimately tied to the presence of boundary conditions (see also Umurhan 2006; Lesur \& Longaretti 2007; Shariff 2009). Johnson \& Gammie (2005a) considered the case of radially stratified disks and analyzed the stability problem for an infinite vertical wavelength at which rotation has no effect because it corresponds to the 2D limiting case (according to the Taylor-Proudmans theorem). They suggested that the aliasing of waves sheared in the right direction to waves sheared in the left direction is responsible for the resurgence of growth (see also Shariff 2009). A similar problem was addressed by Salhi \& Cambon (2010) and a spectral linear theory (SLT) solution was derived for non-axisymmetric disturbances, yielding a general dispersion relation (see their relation (33)). One previous stability study that is related to the work presented here is the radiative instability, since it is due to two helical inertial gravity waves rotating with opposite signs that are emitted by a rotating vortex. This was examined, for instance, in the study by Riedinger et al. (2011).

Other couplings and resonances in stratified, rotating, shearing flows have been shown by others to lead to instabilities. Another related instability is the strato-rotational instability, due to the mode coupling in the case of both rotation and a vertical density stratification (see, Molemaker et al. 2001; Le Bars \& Le Gal 2007). Umurhan (2008) presented a scaling argument that he developed into the shallow water theory of non-axisymmetric disturbances in annular sections of thin Keplerian disks. In that study, it was shown that the strato-rotational instability is contained within generic class of barotropic/baroclinic potential vorticity (PV) instabilities.

Tevzadze et al. (2003) extended the analysis of Chagelishvili et al. (2003) by considering vertically stable, stratified disks under $3 \mathrm{D}$ perturbations. They showed that the combined action of rotation and stratification generates an aperiodic vortex mode undergoing non-modal transient growth. Bodo et al. (2005) performed a detailed analytical and numerical study of the dynamics of perturbations 
(vortex/aperiodic mode, Rossby and spiral-density waves) in 2D compressible disks following Keplerian rotation. In a more recent paper, Tevzadze et al. (2008) focused again on the mathematical and physical aspects of the mode coupling in vertically stable, stratified Keplerian disks. These authors concluded that the bypass concept of turbulence can also be applied to these disks. They investigated the mode coupling in a 2D compressible disk with radial stratification and differential rotation. Tevzadze et al. (2010) also extended once more the same analysis to study the coupling between the vorticity, entropy, and compressional 2D modes. These authors characterized the transient growth in radially stratified disks, with interchanges and mode coupling including a dilatational mode (a new degree of freedom is opened in the system due to compressibility). Also, recently Lesur \& Papaloizou (2010) studied the subcritical (or nonlinear) baroclinic instability in local accretion disks and concluded that such an instability can lead to a weak outward transport of angular momentum due to the generation of density waves by the vortices. This is related to our present work because we are performing here an extension of these previous studies including both radial and vertical stratifications. Above all, we extend the work of Tevzadze et al. (2008) in the same situation by including the transient growth analysis. Indeed, Tevzadze et al. (2008; and previous works by the same authors) have shown that an aperiodic vortex mode is induced by the strato-rotational balance (for a vertical gradient). The vortex mode can generate spiral density waves (or gravito-inertial ones) through linear coupling in shear flows. Both modes (wave and vortex) can extract energy from the background mean flow and exhibit a rapid transient growth behavior due to the non-normality of the shear flow. The vortex can feed the wave but not the opposite. In this paper, we use the SSA and by means of SLT, we study the wave-vortex mode coupling in vertically and radially stratified accretion disks. As in Tevzadze et al. (2008), we introduce a significant simplification by neglecting the rotational-acoustic waves; we assume that their characteristic timescale is much shorter than the vortex and wave modes. This simplification is the Boussinesq approximation used to describe the dynamics of stratified astrophysical disks. We refer the reader to many other related studies for a formal review (see, e.g., Balbus \& Hawley 1998). The main purpose of this work can be summarized as follows.

1. As indicated previously, vertically stable, stratified disks are spectrally stable. This has been proven using a WKB (Wentzel-Kramers-Brillouin) assumption and/or numerical computations (see, e.g., McWilliams \& Yavneh 1998). The proof of such a result by means of Levinson's theorem (see, e.g., Eastham 1989) is given in the present study; this theorem has a wider application since the WKB methods can fail when the timescale for the perturbation is no longer rapid compared with the long wave vector k-changing timescale (see, e.g., Balbus \& Hawley 1992). For this purpose, we introduce a linear response function (or the $3 \times 3$ Green matrix $\mathbf{g}(t)$, which reduces to unity at $t=0$ ). The use of Levinson's theorem requires (1) the solution $\mathbf{g}^{(w)}$ corresponds to a vanishing PV, and (b) the solution $\mathbf{g}^{(v)}$ corresponds to a non-vanishing PV. Accordingly, we recover the relevant decomposition by Chagelishvili et al. (1997) that consists of decomposing the velocity or buoyancy Fourier mode into two parts: a wave mode characterized by a vanishing PV and a vortex mode characterized by a non-vanishing PV: $\mathbf{g}=\mathbf{g}^{(w)}+\mathbf{g}^{(v)}$.

2. In some studies using the SSA (or local Lagrangian coordinates ignoring the boundaries, see, e.g., Afshordi et al. 2005), the case of an infinite vertical wavelength (or $k_{3}=0$ ) at which rotation effects vanish and for which there is a simple analytical solution (see Chagelishvili et al. 1997; Salhi \& Cambon 1997) has been considered to better illustrate the transient growth scenario (see Chagelishvili et al. 2003; Tevzadze et al. 2003; Umurhan \& Regev 2004; Afshordi et al. 2005). More details related to the transient growth rate are presented in Section 5. We show that the transient growth rate in stratified accretion disks under combined vertical and radial stratification is well captured by the dynamics in the $k_{3}=0$ plane provided the ratio of the initial radial wavenumber to the azimuthal wavenumber is large $\left(K_{2} / k_{1} \gg 1\right)$. In most previous studies, initial conditions corresponding to the vortex perturbations in a pure form were considered to compute the transient growth rate. In the present study, we consider initial isotropic conditions with a dense spectrum and we examine in detail the realizability conditions for the choice of the initial total (kinetic+potential) vortex, wave, and mutual energies (i.e., these energies must be positive or zero).

3. Because our SLT analysis is not only "mono-modal" but also involves simultaneously different modes, either from the viewpoint of coupling of modes or from the viewpoint of prediction of statistics from initial disturbances with a dense spectrum, we also analyze the behavior of the power spectrum of the total energy in both the vertical and azimuthal directions. The algebraic form of these spectra is synonymous with particular flow structures such as the streaky structures.

The paper is organized as follows. The governing equations under the Boussinesq approximation are introduced in Section 2. In Section 3, we reduce the number of involved variables by working in the local frame attached to the wave vector, we define the Green's functions for universal initial conditions, and we perform the linear stability of perturbations in the axisymmetric case. In Section 4, we derive the non-normal stability analysis for the vertical stratification case and use Levinson's theorem (to go beyond the WKB analysis) to demonstrate stability. In Section 5, we examine the transient growth of the spectral density of the total (kinetic+potential) energy using the wave-vortex decomposition. In Section 6, we treat, in a similar manner, the general case with combined radial and vertical stratification. Section 7 gives our conclusions.

\section{BOUSSINESQ EQUATIONS}

\subsection{Base Flow}

For the purpose of the linear analysis of stratified thin disks, we employ the shearing sheet formalism in the Boussinesq approximation. The reader may consult Hawley et al. (1995), Balbus (2003), and Regev \& Umurhan (2008) for an extensive discussion of the properties and limitations of the shearing sheet model. In a locally Cartesian coordinate system $\left(x_{1}, x_{2}, x_{3}\right)$ centered at $r=r_{0}$, where $r$ is the radial coordinate, this flow can be approximated as a linear shear in a uniformly rotating frame

$$
\boldsymbol{U}=\left(U_{1}, 0,0\right), \quad U_{1}=A x_{2}, \quad \Omega=\left(0,0, \Omega_{0}\right),
$$

where $A=\left.(r \partial \Omega)\right|_{r=r_{0}}$ and $\Omega_{0}=\Omega\left(r_{0}\right)$. The coordinate system used here differs from the shearing box (SB) convention in that $x_{2}=x_{\mathrm{SB}} \rightarrow\left(r-r_{0}\right)$ and $x_{1}=-y_{\mathrm{SB}} \rightarrow-r_{0}\left(\phi-\phi_{0}\right)$, where $\left(r, \phi, z=x_{3}\right)$ are the cylindrical coordinates. We assume that the 
background buoyancy scalar $\Theta=-\left(g / \rho_{0}\right) \bar{\rho}$ varies linearly in both the radial and vertical directions (see, e.g., Balbus \& Hawley 1991),

$$
\Theta=N_{2}^{2} x_{2}+N_{3}^{2} x_{3}
$$

where $g$ is the gravitational acceleration, $\rho_{0}$ is a reference density, and $\bar{\rho}$ is the background density. Here, $N_{2}^{2}$ and $N_{3}^{2}$ are constants that represent the square of Brunt-Väsäilä frequencies with respect to the $\left(x_{2}, x_{3}\right)$ directions such that (see Salhi et al. 2012)

$$
n_{3} N_{2}^{2}-n_{2} N_{3}^{2}=0, \quad n_{2}^{2}+n_{3}^{2}=1 .
$$

Hence, for stable vertical and radial stratification, $N_{2}^{2}>0$ and $N_{3}^{2}>0$, the unit vector $\boldsymbol{n}$ belongs to the first quadrant of the $\left(x_{2}, x_{3}\right)$ plane, i.e., $n_{2}>0$ and $n_{3}>0$. For $N_{2}^{2}<0$ (unstable radial stratification) and $N_{3}^{2}>0$ (stable vertical stratification), the unit vector $\boldsymbol{n}$ belongs to the fourth quadrant, i.e., $n_{2}>0$ and $n_{3}<0$ (see Figure 1(b)). As indicated by Lesur \& Papaloizou (2010), radially stratified Keplerian disks $\left(A=(3 / 2) \Omega_{0}\right)$ are stable under axisymmetric disturbances provided $(-4 / 9) A^{2}<N_{2}^{2}<0$ (see also Salhi \& Cambon 2010). A general dispersion relation for disks with radial and vertical buoyancy gradients under axisymmetric disturbances is given in Section 3.2.

The flow of Equations (1) and (2) is an exact steady solution of the Boussinesq's equations (written for an inviscid and non-diffusive fluid in Equations (3) and (4)) upon introduction of a base pressure $P\left(x_{2}, x_{3}\right)$, whose gradient balances the Coriolis and buoyancy forces

$$
\begin{gathered}
\nabla \cdot \tilde{\boldsymbol{u}}=0, \quad \partial_{t} \tilde{\boldsymbol{u}}+(\tilde{\boldsymbol{u}} \cdot \nabla) \tilde{\boldsymbol{u}}=-\nabla \tilde{p}-2 \boldsymbol{\Omega} \times \tilde{\boldsymbol{u}}+\tilde{\theta} \boldsymbol{n}, \\
\left(\partial_{t}+\tilde{\boldsymbol{u}} \cdot \nabla\right) \tilde{\theta}=0 .
\end{gathered}
$$

$\tilde{p}$ is the pressure divided by $\rho_{0}$ and includes the centrifugal potential. $\boldsymbol{n}=\left(0, n_{2}, n_{3}\right)^{T}$ denotes a fixed unit vector antiparallel to the gravitational acceleration vector $\boldsymbol{g}$. The viscosity effect will be addressed later considering, for simplicity, the case where the Prandtl number (i.e., the ratio of diffusivity to kinematic viscosity) is unity.

\subsection{Linearized Equations for the Disturbances}

Following standard methods, the base state velocity is $\boldsymbol{U}=\mathbf{A} \cdot \boldsymbol{x}$, where $A_{i j}=A \delta_{i 1} \delta_{j 2}$. The pressure $P\left(x_{2}, x_{3}\right)$ and buoyancy scalar $\Theta\left(x_{2}, x_{3}\right)$ are perturbed by adding an infinitesimal disturbance $\boldsymbol{u}(\boldsymbol{x}, t), p(\boldsymbol{x}, t)$, and $\theta(\boldsymbol{x}, t)$. The linearized equations of motion for the disturbance are

$$
\begin{gathered}
\nabla \cdot \boldsymbol{u}=0, \quad D_{t} \boldsymbol{u}=-\mathbf{A} \cdot \boldsymbol{u}-2 \Omega \boldsymbol{n} \times \boldsymbol{u}-\nabla p+\theta \boldsymbol{n}, \\
D_{t} \theta=-\left(N_{2}^{2} u_{2}+N_{3}^{2} u_{3}\right), \quad n_{3} N_{2}^{2}=n_{2} N_{3}^{2},
\end{gathered}
$$

where $D_{t}(\cdot)=\left(\partial_{t}+S x_{2} \partial_{x_{1}}\right)(\cdot)$. Disturbances are sought in terms of advected Fourier modes (see, e.g., Moffatt 1967; Craik 1989), also called Kelvin modes or shear waves,

$$
(\boldsymbol{u}(\boldsymbol{x}, t), p(\boldsymbol{x}, t), \theta(\boldsymbol{x}, t))=(\hat{\boldsymbol{u}}(\boldsymbol{k}, t), \hat{p}(\boldsymbol{k}, t), \hat{\theta}(\boldsymbol{k}, t)) \exp (\iota \boldsymbol{k}(t) \cdot \boldsymbol{x}),
$$

where the components of the time-dependent wave vector $\boldsymbol{k}(t)$ are of the form (see Moffatt 1967)

$$
k_{1}=K_{1} \quad k_{2}(t)=K_{2}-K_{1} A t, \quad k_{3}=K_{3} .
$$

The capital letters denote initial values: $\boldsymbol{K}=\boldsymbol{k}(t=0)$. The substitution of the solution (6) into the system (5) yields the following differential system:

$$
\begin{gathered}
\boldsymbol{k} \cdot \hat{\boldsymbol{u}}=0, \quad \dot{\hat{\boldsymbol{u}}}=-\mathbf{A} \cdot \hat{\boldsymbol{u}}-2 \Omega \boldsymbol{n} \times \hat{\boldsymbol{u}}-\imath \hat{p} \boldsymbol{k}+\hat{\theta} \boldsymbol{n}, \\
\dot{\hat{\theta}}=-\left(N_{2}^{2} \hat{u}_{2}+N_{3}^{2} \hat{u}_{3}\right), \quad n_{3} N_{2}^{2}=n_{2} N_{3}^{2} .
\end{gathered}
$$

In addition, the above system admits a temporal invariant from the conservation of absolute PV (Ertel theorem). Conservation of the corresponding full quadratic term $(\nabla \times \tilde{\boldsymbol{u}}) \cdot(\nabla \tilde{\theta})$ results from Equations (3) and (4), so that the linear perturbation of the PV is

$$
\tilde{\pi}(\boldsymbol{x}, t)=(2 \Omega-A) \frac{\partial \theta}{\partial x_{3}}+N_{2}^{2} \omega_{2}+N_{3}^{2} \omega_{3},
$$

where $\omega=\boldsymbol{\nabla} \times \boldsymbol{u}$ is the vorticity disturbance and $2 \Omega-A$ is the (sole) vertical component of the absolute vorticity of the base flow. Its Fourier counterpart, as in Equation (6), is

$$
\hat{\pi}=\imath(2 \Omega-A) k_{3} \hat{\theta}+\imath N_{2}^{2}\left(k_{3} \hat{u}_{1}-k_{1} \hat{u}_{3}\right)+\imath N_{3}^{2}\left(k_{1} \hat{u}_{2}-k_{2} \hat{u}_{1}\right) .
$$

It should be noted that in the magnetized stratified disks, $\tilde{\pi}(\boldsymbol{x}, t)$ does not constitute a Lagrangian invariant, while its counterpart, i.e., the magnetic potential induction $\tilde{\pi}_{m}=\tilde{\boldsymbol{b}} \cdot \nabla \tilde{\theta}$, where $\tilde{\boldsymbol{b}}$ is the magnetic field, constitutes a Lagrangian invariant for an inviscid and non-diffusive fluid as shown recently by Salhi et al. (2012). 


\section{THE REDUCED SYSTEM AND A SIMPLIFIED STABILITY ANALYSIS}

\subsection{Toroidal, Poloidal, and Buoyancy Modes}

A way to reduce the number of components in the linear system of equations is to use a local orthonormal frame $\left(\boldsymbol{e}^{(1)}, \boldsymbol{e}^{(2)}, \boldsymbol{e}^{(3)}\right)$, where $\boldsymbol{e}^{(3)}=\boldsymbol{k} / k$ and

$$
\boldsymbol{e}^{(1)}=\left(\frac{k_{2}}{k_{h}},-\frac{k_{1}}{k_{h}}, 0\right)^{T}, \quad \boldsymbol{e}^{(2)}=\left(\frac{k_{1} k_{3}}{k_{h} k}, \frac{k_{2} k_{3}}{k_{h} k},-\frac{k_{h}}{k}\right)^{T} .
$$

In this frame, the velocity field is decomposed into toroidal $u^{(1)}$ and poloidal $u^{(2)}$ components as $\hat{\boldsymbol{u}}=u^{(1)} \boldsymbol{e}^{(1)}+u^{(2)} \boldsymbol{e}^{(2)}$ (see, e.g., Salhi et al. 2012; Chandrasekhar 1961). For consistency, the buoyancy variable $\hat{\theta}$ is scaled to have the same dimension as the velocity modes

$$
u^{(1)}=\frac{k_{2}}{k_{h}} \hat{u}_{1}-\frac{k_{1}}{k_{h}} \hat{u}_{2}, \quad u^{(2)}=-\frac{k}{k_{h}} \hat{u}_{3}, \quad u^{(3)} \equiv-\frac{1}{A R_{i}} \hat{\theta},
$$

where $k_{h}=\sqrt{k_{1}^{2}+k_{2}^{2}}$ is the horizontal wavenumber and $R_{i}$ is the Richardson number that compares the shear timescale to the buoyancy timescale (see, e.g., Drazin $\&$ Reid 1981). $R_{i}$ is defined here as

$$
R_{i}=N_{3}^{2} /\left(n_{3} A^{2}\right)=N_{2}^{2} /\left(n_{2} A^{2}\right)
$$

since we consider stable radial and vertical stratification. The linear differential system for the vector $\boldsymbol{v}$ of toroidal, poloidal, and potential components is

$$
\frac{d \boldsymbol{v}}{d \tau}=\mathbf{L} \cdot \boldsymbol{v}
$$

deduced from the system (8), where $\tau=A t$ is the dimensionless time. The three-rank matrix $\mathbf{L}$ is of the form

$$
\mathbf{L}=\left[\begin{array}{ccc}
\frac{k_{1} k_{2}}{k_{h}^{2}} & -\left(1+R_{\Omega}\right) \frac{k_{3}}{k} & R_{i}\left(n_{2} \frac{k_{1}}{k_{h}}\right) \\
\left(R_{\Omega}+2 \frac{k_{1}^{2}}{k_{h}^{2}}\right) \frac{k_{3}}{k} & -\frac{k_{1} k_{2}}{k_{h}^{2}} \frac{k_{3}^{2}}{k^{2}} & R_{i}\left(n_{3} \frac{k_{h}}{k}-n_{2} \frac{k_{2} k_{3}}{k_{h} k}\right) \\
-n_{2} \frac{k_{1}}{k_{h}} & -n_{3} \frac{k_{h}}{k}+n_{2} \frac{k_{2} k_{3}}{k_{h} k} & 0
\end{array}\right],
$$

in which

$$
R_{\Omega}=\frac{-2 \Omega_{0}}{A}=\frac{2 \Omega_{0}}{\left(r \frac{d \Omega}{d r}\right)_{r=r_{0}}}=-\frac{2}{q}
$$

is the rotation number ( $R_{\Omega}=-4 / 3$ for Keplerian disks). More details of the derivation of system (12) are given in Salhi et al. (2012). These authors studied the effect of radial and vertical stratification on the MRI growth rate. In addition, it is more convenient to introduce the Green's function $\mathbf{g}$ such that $\boldsymbol{v}(\boldsymbol{k}, t)=\mathbf{g} \cdot \boldsymbol{v}(\boldsymbol{K}, 0)$. This function is governed by the same equation as $\boldsymbol{v}$, i.e.,

$$
\frac{d \mathbf{g}}{d \tau}=\mathbf{L} \cdot \mathbf{g}
$$

but with the universal initial condition $\mathbf{g}(0)=\mathbf{I}_{3}$, where $\mathbf{I}_{3}$ is the three-rank unit matrix. The above $3 \mathrm{D}$ system can be reduced to a $2 \mathrm{D}$ one if we use the conservation of the PV defined by Equation (9), which can be rewritten in terms of the toroidal-poloidal-buoyancy mode:

$$
q_{0} \equiv \iota \frac{n_{3}}{N_{3}^{2}} \hat{\pi}=\left(n_{3} k_{h}-n_{2} \frac{k_{2} k_{3}}{k_{h}}\right) u^{(1)}-n_{2} \frac{k_{1} k}{k_{h}} u^{(2)}-\left(1+R_{\Omega}\right) k_{3} u^{(3)} .
$$

Or, equivalently,

$$
\begin{aligned}
& \left(n_{3} k_{h}-n_{2} \frac{k_{2} k_{3}}{k_{h}}\right) g_{1 j}-n_{2} \frac{k_{1} k}{k_{h}} g_{2 j}-\left(1+R_{\Omega}\right) k_{3} g_{3 j} \\
& \quad=\left[1-\delta\left(q_{0}\right)\right]\left[\left(n_{3} K_{h}-n_{2} \frac{K_{2} k_{3}}{K_{h}}\right) \delta_{1 j}-n_{2} \frac{k_{1} K}{K_{h}} \delta_{2 j}-\left(1+R_{\Omega}\right) k_{3} \delta_{3 j}\right],
\end{aligned}
$$

where $\delta\left(q_{0}\right)=1$ if the constant $q_{0}$ is zero and $\delta\left(q_{0}\right)=0$ if $q_{0} \neq 0$. Reduction of the linear system in Equation (14) to a rank-two non-homogeneous system, using Equation (16), will be used in Section 4. 


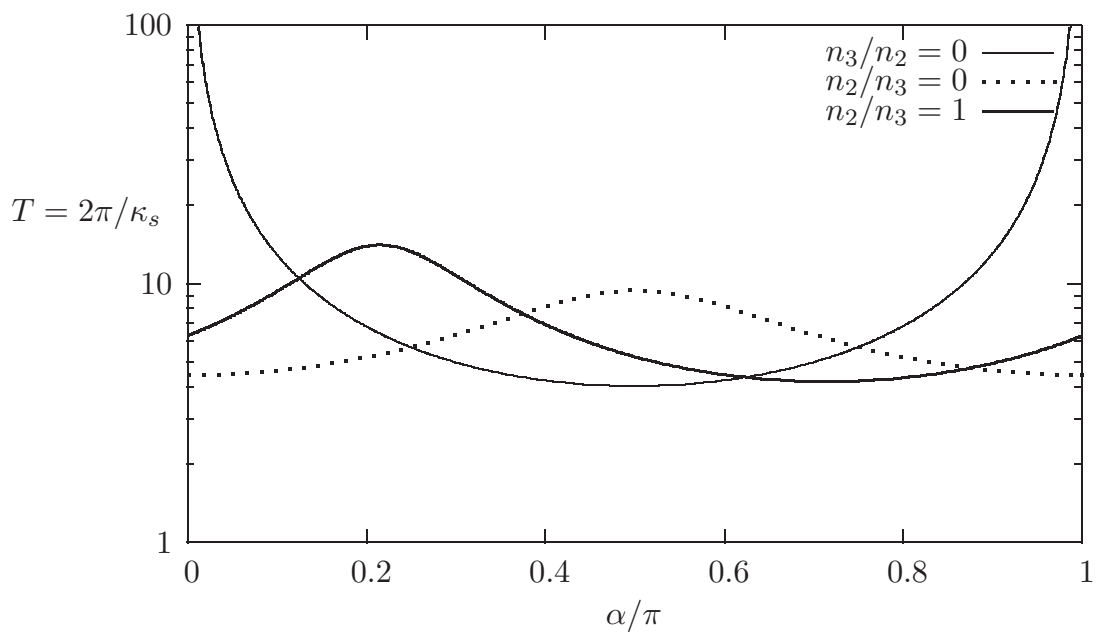

Figure 2. Inertia-gravity-shear waves in the $k_{1}=0$ plane. The period of oscillation vs. the angle $\alpha$ (i.e., the angle between the wave vector and the radial direction) is shown for the case of a Keplerian disk with vertical stratification $\left(n_{2}=0, n_{3}=1\right.$ and $\left.R_{i}=1\right)$, radial stratification $\left(n_{3}=0, n_{2}=1\right.$, and $\left.R_{i}=1\right)$, or combined radial and vertical stratification $\left(n_{2}=n_{3}=\sqrt{2} / 2\right.$ and $\left.R_{i}=1\right)$.

\subsection{Simplified Stability Analysis}

Under axisymmetric disturbances (i.e., $k_{1}=0$ ), the wave vector $\boldsymbol{k}$, as well as the matrix $\mathbf{L}$, becomes time-independent. In that case, one easily determines the eigenvalues of the matrix $\mathbf{L}$. There are solutions of the algebraic equation $\operatorname{Det}\left(\mathbf{L}-\lambda \mathbf{I}_{3}\right)=0$, or equivalently, $\lambda\left(\lambda^{2}-\kappa_{s}^{2} / A^{2}\right)=0$, where

$$
\kappa_{s}^{2}=\kappa^{2} \sin ^{2} \alpha+\frac{N_{3}^{2}}{n_{3}} \cos ^{2} \gamma, \quad \kappa^{2}=2 \Omega_{0}\left(2 \Omega_{0}-A\right),
$$

with

$$
\cos \gamma=n_{3} \frac{k_{h}}{k}-n_{2} \frac{k_{2} k_{3}}{k_{h} k}=n_{3} \cos \alpha-n_{2} \sin \alpha .
$$

$\alpha$ is the angle between the wave vector and the transverse (radial) direction, such that $\cos \alpha=k_{2} / k$. We note that, for radially stratified disks, the dispersion relation (17) reduces to the Solberg-Hoiland criterion, i.e., $\kappa^{2}+N_{2}^{2}>0$ for stability.

As stressed in Section 1, the angular velocity in an incompressible uniform disk can be described by $\Omega(r)=\Omega_{0}\left(r_{0} / r\right)^{q}$, where $q=3 / 2$ for a Keplerian disk. Hence, the square of the epicyclic frequency takes the form $\kappa^{2}=2(2-q) \Omega_{0}^{2}$, which has a positive sign indicating stability except for a disk with a constant specific angular momentum $(q=2)$, for which it vanishes $(\kappa=0)$. Under stable stratification, i.e., $N_{3}^{2} \geqslant 0$ and $n_{3} \geqslant 0$ (see Section 2.1), the disk remains stable $\left(\kappa_{s}^{2} \geqslant \kappa^{2}>0\right)$, implying that the period of the inertia-shear-gravity waves $\left(T=2 \pi / \kappa_{s}\right)$ is less than or equal to the period of the inertia-shear waves propagating in the $k_{1}=0$ plane with the phase velocity $\boldsymbol{V}_{\varphi}=\left(\kappa_{s} / k^{2}\right) \boldsymbol{k}$. Figure 2 shows the variation of the period $T=2 \pi / \kappa_{s}$ of the wave versus the angle $\alpha$ for stratified Keplerian disks with $R_{i}=1$. Three cases are considered: radial stratification only $\left(N_{3}=0\right)$, vertical stratification only $\left(N_{2}=0\right)$, and combined radial and vertical stratification $\left(n_{2} / n_{3}=1\right)$. As can be seen, the waves are more dispersive in the radially stratified case than in the vertically stratified one.

Disks with unstable radial stratification and stable vertical stratification $\left(N_{2}^{2}<0\right.$ and $n_{2}>0, N_{3}^{2}>0$ and $\left.n_{3}<0\right)$ are stable provided that

$$
-\kappa^{2} \sin ^{2} \alpha<\left(N_{2}^{2} / n_{2}\right) \cos ^{2} \gamma<0 .
$$

This implies that $-\Omega_{0}^{2}<N_{2}^{2}<0$ for unstably radially stratified Keplerian disks (without vertical stratification). Details of the unstable domain, in terms of $R_{\Omega}, n_{3}, n_{2}$ and $\alpha$, are easily derived from Equation (17) and are not given here for the sake of brevity; our focus is on Keplerian disks and on the non-axisymmetric modes in them.

As a final remark on the axisymmetric case $k_{1}=0$, the use of the $q_{0}$ invariant holds, and a non-homogeneous linear system of two equations is still valid. However, in the stable case, one recovers only a simple superposition of periodic inertia-gravity waves and a constant vortex mode, which is the quasi-geostrophic part of the disturbance flow without mean shear (details can be found in Appendix A.4). In this case, the presence of the shear is marginal, and only the non-axisymmetric case is essential for a dramatic transient growth fed by the vortex mode.

Under non-axisymmetric disturbances, the use of a WKB-type of analysis (see, e.g., McWilliams \& Yavneh 1998), which consists of replacing the time-dependent coefficients in Equation (13) with their long-time limits, gives stability. In fact, the long-time limit of the matrix $\mathbf{L}$ is of the form

$$
\mathbf{L}_{\infty}=\left(\begin{array}{ccc}
0 & 0 & 0 \\
0 & 0 & n_{3} R_{i} \\
0 & -n_{3} & 0
\end{array}\right),
$$


with eigenvalues $\lambda_{1,2}= \pm ı n_{3} \sqrt{R_{i}}$ indicating stability independent of rotation provided that $R_{i}>0$.

Because WKB methods can break down when the timescale for the perturbation evolution is no longer rapid compared with the long $\boldsymbol{k}$-changing timescale (see, e.g., Balbus \& Hawley 1992), we propose to rigorously demonstrate the stability of vertically stratified disks (referred to as VSD) under non-axisymmetric disturbances (see Section 4).

\section{SINGLE-MODE NON-NORMAL STABILITY ANALYSIS FOR VSD}

We will now use the explicit reduction of the rank of the linear system of equations from three to two. We use the conservation of linearized absolute PV. As stressed before, this reduction is only relevant for the non-axisymmetric mode. For $k_{1} \neq 0$ indeed, one recovers the time dependence of the wavevector in Equation (7): this time dependency in SLT reflects the explicit advection by the base shear flow, which renders the non-normality of the linear operator.

When there is no radial stratification $\left(n_{2}=0, n_{3}=1\right)$, the relation (16) characterizing the conservation of the PV reduces to

$$
g_{1 j}=\left(1+R_{\Omega}\right) \frac{k_{3}}{k_{h}} g_{3 j}+\left[1-\delta\left(q_{0}\right)\right]\left[\frac{K_{h}}{k_{h}} \delta_{1 j}-\left(1+R_{\Omega}\right) \frac{k_{3}}{k_{h}} \delta_{3 j}\right] .
$$

The substitution of the above relation into the system (14) leads to the following 2D nonhomogeneous system (see Appendix A.1):

$$
\frac{d}{d \tau}\left(\begin{array}{c}
\frac{k}{k_{h}} g_{2 j} \\
g_{3 j}
\end{array}\right)-\left(\begin{array}{cc}
0 & C_{12}(\tau) \\
C_{21}(\tau) & 0
\end{array}\right) \cdot\left(\begin{array}{c}
\frac{k}{k_{h}} g_{2 j} \\
g_{3 j}
\end{array}\right)=\left(\begin{array}{c}
{\left[1-\delta\left(q_{0}\right)\right] h_{j}(\tau)} \\
0
\end{array}\right),
$$

where

$$
\begin{gathered}
C_{12}(\tau)=\left(1+R_{\Omega}\right)\left(R_{\Omega}+2 \frac{k_{1}^{2}}{k_{h}^{2}}\right) \frac{k_{3}^{2}}{k_{h}^{2}}+R_{i}, \\
C_{21}(\tau)=-\frac{k_{h}^{2}}{k^{2}}=-1+\frac{k_{3}^{2}}{k^{2}}, \\
h_{j}(\tau)=\left(R_{\Omega}+2 \frac{k_{1}^{2}}{k_{h}^{2}}\right) \frac{k_{3}}{k_{h}}\left[\frac{K_{h}}{k_{h}} \delta_{1 j}-\left(1+R_{\Omega}\right) \frac{k_{3}}{k_{h}} \delta_{3 j}\right],
\end{gathered}
$$

where $j=1,2,3$. We first consider the homogeneous system, i.e., we set $\delta\left(q_{0}\right)=1$ in Equation (20). With the help of the following theorem, we will rigorously demonstrate the stability of that system.

Theorem 1 (Levinson, see Lagnado et al. 1984; Eastham 1989): Let the linear differential system $d \boldsymbol{X} / d \tau=\left(\mathbf{C}_{\mathbf{0}}+\mathbf{C}_{1}(\tau)\right) \cdot \boldsymbol{X}$, where $\mathbf{C}_{0}$ is an $n \times n$ constant matrix with $n$ distinct eigenvalues $\lambda_{1}, \lambda_{2}, \ldots, \lambda_{n}$; and $\mathbf{C}_{1}(\tau)$ is an $n \times n$ matrix for which the elements $\left(\mathbf{C}_{1}\right)_{i j}$ satisfy

$$
\int_{0}^{\infty}\left|\left(\mathbf{C}_{1}\right)_{i j}(\tau)\right| d \tau<\infty \quad(i, j=1,2, \ldots, n) .
$$

Then, for large $\tau$, there exist $n$ independent solutions $\boldsymbol{X}^{(1)}, \boldsymbol{X}^{(2)}, \ldots ., \boldsymbol{X}^{(n)}$ such that as $\tau \rightarrow \infty$

$$
\boldsymbol{X}^{(\ell)}(\tau)=\left[\boldsymbol{Y}^{(\ell)}+\mathcal{O}(1)\right] \exp \left(\lambda_{\ell} \tau\right) \quad(\ell=1,2, \ldots, n),
$$

where $\boldsymbol{Y}^{(\ell)}(1 \leqslant \ell \leqslant n)$ is an eigenvector of $\mathbf{C}_{0}$ corresponding to the eigenvalue $\lambda_{\ell}$.

Accordingly, we decompose the matrix $\mathbf{C}(\tau)$ into two matrices, $\mathbf{C}=\mathbf{C}_{0}+\mathbf{C}_{1}(\tau)$, where $\mathbf{C}_{0}$ is a constant matrix and $\mathbf{C}_{1}(\tau)$ is a time-dependent one,

$$
\mathbf{C}_{0}=\left(\begin{array}{cc}
0 & R_{i} \\
-1 & 0
\end{array}\right), \quad \mathbf{C}_{1}(\tau)=\left(\begin{array}{cc}
0 & C_{12}-R_{i} \\
k_{3}^{2} / k^{2} & 0
\end{array}\right),
$$

and we verify that the eigenvalues of the matrix $\mathbf{C}_{0}$ are distinct: $\lambda_{1,2}= \pm l \sqrt{R_{i}}$. Moreover, we show that condition (22) stated in Theorem 1 is satisfied (see Appendix A.2)

$$
\int_{0}^{\infty}\left|\mathbf{C}_{1}(\tau)\right| d \tau<\infty
$$

It follows that the solution of the homogeneous system exhibits an oscillatory behavior for long times. With the aid of the following theorem, we will now demonstrate that the nonhomogeneous system (20) is stable.

Theorem 2: (see Struble 1962, p. 119): If all solutions of $d \boldsymbol{X} / d \tau=\left(\mathbf{C}_{0}+\mathbf{C}_{1}(\tau)\right) \cdot \boldsymbol{X}$ are bounded as $\tau \rightarrow \infty$, then the same is true of the inhomogeneous system $d \boldsymbol{X} / d \tau=\left(\mathbf{C}_{0}+\mathbf{C}_{1}(\tau)\right) \cdot \boldsymbol{X}+\boldsymbol{h}(\tau)$ provided condition (22) in Theorem 1 is satisfied and $\boldsymbol{h}(\tau)$ is impulsively small as $\tau \rightarrow \infty$,

$$
\int_{0}^{\infty}|\boldsymbol{h}(\tau)| d \tau<\infty .
$$


As shown previously, the homogeneous system satisfies condition (22) in Theorem 1. In addition, we show in Appendix A.2 that the nonhomogeneous term (i.e., the term $h_{j}(\tau)$ in (20)) satisfies the condition (26). Accordingly, we may conclude that a disk under stable vertical stratification $\left(N_{3}^{2}>0\right)$ is stable under non-axisymmetric disturbances.

We will now show that the above proof of stability can allow us to recover the relevant decomposition first proposed by Chagelishvili et al. (1997). By setting $\delta\left(q_{0}\right)=1$ in system (20), we obtain its associated homogeneous system. We denote by $g_{i j}^{(w)}(i=2,3, j=1,2,3)$ the solution of that system, or equivalently, the solution at vanishing PV. We note that, at vanishing PV, Equation (19) reduces to

$$
g_{1 j}^{(w)}(t)=\left(1+R_{\Omega}\right) \frac{k_{3}}{K_{h}} g_{3 j}^{(w)}(t)
$$

allowing us to determine $g_{1 j}^{(w)}$ from the solution $g_{3 j}^{(w)}$. In a similar manner, we denote by $g_{i j}^{(v)}(i=2,3, j=1,2,3)$ a particular solution of the nonhomogeneous system (20), and from Equation (19) we express the component $g_{1 j}^{(v)}$ in terms of $g_{3 j}^{(v)}$,

$$
g_{1 j}^{(w)}(t)=\left(1+R_{\Omega}\right) \frac{k_{3}}{K_{h}} g_{3 j}^{(w)}(t)-\left[\delta_{1 j}-\left(1+R_{\Omega}\right) \frac{k_{3}}{K_{h}} \delta_{3 j}\right] \quad(j=1,2,3)
$$

since the particular solution corresponds to a nonzero value for the PV (i.e., $q_{0} \neq 0$ and hence $\delta\left(q_{0}\right)=0$ ). Accordingly, at any time $t$, the complete solution $g_{i j}(t)$ is the sum of $g_{i j}^{(w)}$ and $g_{i j}^{(v)}$,

$$
g_{i j}(t)=g_{i j}^{(w)}(t)+g_{i j}^{(v)}(t)
$$

Then, we may introduce the wave and vortex modes as

$$
u_{w}^{(i)}(t)=g_{i j}^{(w)}(t) u_{0}^{(j)}, \quad u_{v}^{(i)}(t)=g_{i j}^{(v)}(t) u_{0}^{(j)}
$$

Therefore, we recover the relevant decomposition first proposed by Chagelishvili et al. (1997),

$$
u^{(i)}(t)=g_{i j}(t) u_{0}^{(j)}=\underbrace{u_{w}^{(i)}(t)}_{\text {wave }\left(q_{0}=0\right)}+\underbrace{u_{v}^{(i)}(t)}_{\operatorname{vortex}\left(q_{0} \neq 0\right)} .
$$

As for the initial condition for $g_{i j}^{(w)}$ and $g_{i j}^{(v)}$, we choose the following simple form that is justified in Appendix A.5:

$$
g_{i j}^{(w)}(0)=\left(\begin{array}{ccc}
0 & 0 & \left(1+R_{\Omega}\right) \frac{k_{3}}{K_{h}} \\
0 & 1 & 0 \\
0 & 0 & 1
\end{array}\right) \quad g_{i j}^{(v)}(0)=\left(\begin{array}{ccc}
1 & 0 & -\left(1+R_{\Omega}\right) \frac{k_{3}}{K_{h}} \\
0 & 0 & 0 \\
0 & 0 & 0
\end{array}\right)
$$

leading to

$$
g_{11}^{(w)}=g_{21}^{(w)}=g_{31}^{(w)}=0, \quad g_{12}^{(v)}=g_{22}^{(v)}=g_{32}^{(v)}=0
$$

at any time (see Appendix A.3). The interplay between disk rotation and stratification gives rise to the vortex/aperiodic mode, characterized by $g_{i j}^{(v)}$, that is able to extract the basic flow energy transiently (see Tevzadze et al. 2003, 2008). The spiral-density wave mode is characterized by $g_{i j}^{(w)}$.

When $k_{3} \neq 0$ and $k_{1} \neq 0$, the time evolution of $g_{i j}^{(w)}$ and $g_{i j}^{(v)}$ for a given $\boldsymbol{k} / \boldsymbol{k}$ in the case of vertically stable, stratified Keplerian disks $\left(R_{\Omega}=-4 / 3\right)$ is determined numerically. Due to the above initial condition, the determination of energies in $\boldsymbol{k}$ space, which allows us to estimate the transient growth rate as well as the one-dimensional spectra, requires the integration of the following reduced differential system deduced from the system (20):

$$
\frac{d}{d \tau}\left(\begin{array}{cc}
\left(\frac{k}{k_{h}} g_{21}^{(v)}\right. & \left(\begin{array}{c}
\frac{k}{k_{h}} g_{22}^{(w)}
\end{array}\right) \\
g_{31}^{(v)} & g_{32}^{(w)}
\end{array}\right)=\left(\begin{array}{cc}
0 & C_{12} \\
C_{21} & 0
\end{array}\right) \cdot\left(\begin{array}{c}
\left(\frac{k}{k_{h}} g_{21}^{(v)}\right. \\
g_{31}^{(v)}
\end{array}\right)\left(\begin{array}{c}
\frac{k}{k_{h}} g_{22}^{(w)} \\
g_{32}^{(w)}
\end{array}\right)+\left(\begin{array}{cc}
h_{1} & 0 \\
0 & 0
\end{array}\right),
$$

with the initial condition

$$
g_{22}^{(w)}(0)=1, \quad g_{21}^{(v)}(0)=g_{31}^{(v)}(0)=g_{32}^{(w)}(0)=0,
$$

where $C_{12}, C_{21}$, and $h_{1}$ are given by Equation (21). A fourth-order Runge-Kutta scheme with a time step of $\delta \tau=10^{-3}$ has been used to perform the numerical integration of the above system. Note that the choice of this initial condition, which ensures the realizability conditions, gives rise to a zero value for the mutual energy, i.e., the energy characterizing the interaction between the vortex and wave modes (see Appendix A.5). 


\section{TRANSIENT GROWTH OF ENERGIES IN VSD}

While the linear analysis shows that the unmagnetized disks are spectrally stable (i.e., there are no exponentially growing solutions) with or without stable stratification, as rigorously shown in the previous two sections, there is irrefutable observational evidence that Keplerian disks have to be turbulent. This fact incited many authors to adopt the bypass concept of turbulence for these disks (see, e.g., Ioannou \& Kakouris 2001; Chagelishvili et al. 2003, Tevzadze et al. 2003, 2008; Umurhan \& Regev 2004; Afshordi et al. 2005). This concept has been developed by the hydrodynamic community for spectrally stable shear flows, as indicated in Section 1 . In the bypass concept, perturbations undergo a transient growth. If they have an initially finite amplitude, they may reach an amplitude that is sufficiently large to allow positive feedback through nonlinear interactions that repopulate the growing disturbances. This mechanism could plausibly sustain turbulence for large enough Reynolds numbers.

By setting $v=\left(u^{(1)}, u^{(2)}, \sqrt{R_{i}} u^{(3)}\right)^{T}$, the spectral density of the total energy can be expressed as the scalar product

$$
2 \mathcal{E}_{T}(\tau)=\langle\boldsymbol{v}(\tau), \boldsymbol{v}(\tau)\rangle=\langle\mathbf{g} \cdot \boldsymbol{v}(0), \mathbf{g} \cdot \boldsymbol{v}(0)\rangle=\left\langle\boldsymbol{v}(0), \mathbf{g}^{A} \mathbf{g} \cdot \boldsymbol{v}(0)\right\rangle,
$$

where $\mathbf{g}^{A}=\mathbf{g}^{T *}$ is the transconjugate matrix of $\mathbf{g}$ and the maximum energy growth $G(\tau)$ obtainable at time $\tau$ over all possible initial conditions $\hat{\boldsymbol{v}}(0)$ is (see, e.g., Blackburn et al. 2008)

$$
G(\tau)=\max _{\boldsymbol{v}(0)} \frac{\left\langle\boldsymbol{v}(0), \mathbf{g}^{A} \mathbf{g} \cdot \boldsymbol{v}(0)\right\rangle}{\langle\boldsymbol{v}(0), \boldsymbol{v}(0)\rangle} .
$$

In some studies, the Rayleigh quotient,

$$
\mathcal{R}_{a}=\frac{\left\langle\boldsymbol{v}(0), \mathbf{g}^{A} \mathbf{C g} \cdot \boldsymbol{v}(0)\right\rangle}{\left\langle\boldsymbol{v}(0), \mathbf{g}^{A} \mathbf{g} \cdot \boldsymbol{v}(0)\right\rangle}
$$

is also used to characterize the transient growth (see, e.g., Brandenburg \& Dintrans 2006).

In the present study, we restrict our attention to particular initial conditions corresponding to initial isotropic conditions with zero helicity, zero initial potential energy, and zero initial density fluxes (more details are reported in Appendix A.5):

$$
\begin{gathered}
\left\langle u_{0}^{(1) *} u_{0}^{(1)}\right\rangle=\left\langle u_{0}^{(2) *} u_{0}^{(2)}\right\rangle=\mathcal{E}_{\kappa}(0), \\
\left\langle u_{0}^{(3) *} u_{0}^{(3)}\right\rangle=0, \quad\left\langle u_{0}^{(i) *} u_{0}^{(j)}\right\rangle=0(i \neq j)
\end{gathered}
$$

$i, j=1,2,3$, so that $\mathcal{E}_{T}(0)=\mathcal{E}_{\kappa}(0)$. Thus, the evaluation of the growth rate $G(\tau)$ requires the determination of the total (kinetic+potential) energy $\mathcal{E}_{T}(t)$ in $\boldsymbol{k}$ space. This total energy can be expressed in terms of the components of the matrix $\mathbf{g}$ (see Appendix A.5):

$$
\frac{\mathcal{E}_{T}(t)}{\mathcal{E}_{T}(0)}=\frac{1}{2} \sum_{i=1}^{2} \sum_{j=1}^{2}\left|g_{i j}\right|^{2}+\frac{R_{i}}{2} \sum_{j=1}^{2}\left|g_{3 j}\right|^{2} .
$$

Some theoretical insights in characterizing the role of the vortex and wave modes in the transient growth can be gained by substituting the solutions $g_{i j}^{(w)}$ and $g_{i j}^{(v)}$ into Equation (34). This defines the so-called wave and vortex energies

$$
\begin{gathered}
\frac{\mathcal{E}_{T}^{(w)}(t)}{\mathcal{E}_{T}(0)}=\frac{1}{2}\left|g_{22}^{(w)}\right|^{2}+\frac{1}{2}\left(R_{i}+\left(1+R_{\Omega}\right)^{2} \frac{k_{3}^{2}}{k_{h}^{2}}\right)\left|g_{32}^{(w)}\right|^{2}, \\
\frac{\mathcal{E}_{T}^{(v)}(t)}{\mathcal{E}_{T}(0)}=\underbrace{\left(1+R_{\Omega}\right) \frac{k_{3} K_{h}}{k_{h}^{2}} \Re g_{31}^{(v)}}_{(I)}+\underbrace{\frac{1}{2} \frac{K_{h}^{2}}{k_{h}^{2}}}_{(I I)}+\underbrace{\frac{1}{2}\left[\left(R_{i}+\left(1+R_{\Omega}\right)^{2} \frac{k_{3}^{2}}{k_{h}^{2}}\right)\left|g_{31}^{(v)}\right|^{2}+\left|g_{21}^{(v)}\right|^{2}\right]}_{(I I I)} .
\end{gathered}
$$

Under the initial conditions described by Equation (31), the "mutual" energy $\mathcal{E}_{T}^{(v w)}(t)$ —or equivalently, the energy characterizing the interaction between the vortex and the wave modes - is zero at any time (see Appendix A.5) so that $\mathcal{E}_{T}(t)=\mathcal{E}_{T}^{(w)}(t)+\mathcal{E}_{T}^{(v)}(t)$.

The case of an infinite vertical wavelength (i.e., $k_{3}=0$ ) for which a simple analytical solution is found (see Appendix A.4) is considered by many authors to illustrate the transient growth rate (Chagelishvili et al. 2003; Umurhan \& Regev 2004; Afshordi et al. 2005). In that case, both terms (I) and (III) in Equation (36) vanish, so that the "vortex" energy behaves like $K_{h}^{2} / k_{h}^{2}$,

$$
\frac{\mathcal{E}_{T}^{(v)}\left(k_{3}=0, t\right)}{\mathcal{E}_{T}(0)}=\frac{1}{2} \frac{K_{h}^{2}}{k_{h}^{2}}=\frac{1}{2} \frac{k_{1}^{2}+K_{2}^{2}}{\left(k_{1}^{2}+\left(K_{2}-k_{1} \tau\right)^{2}\right)},
$$




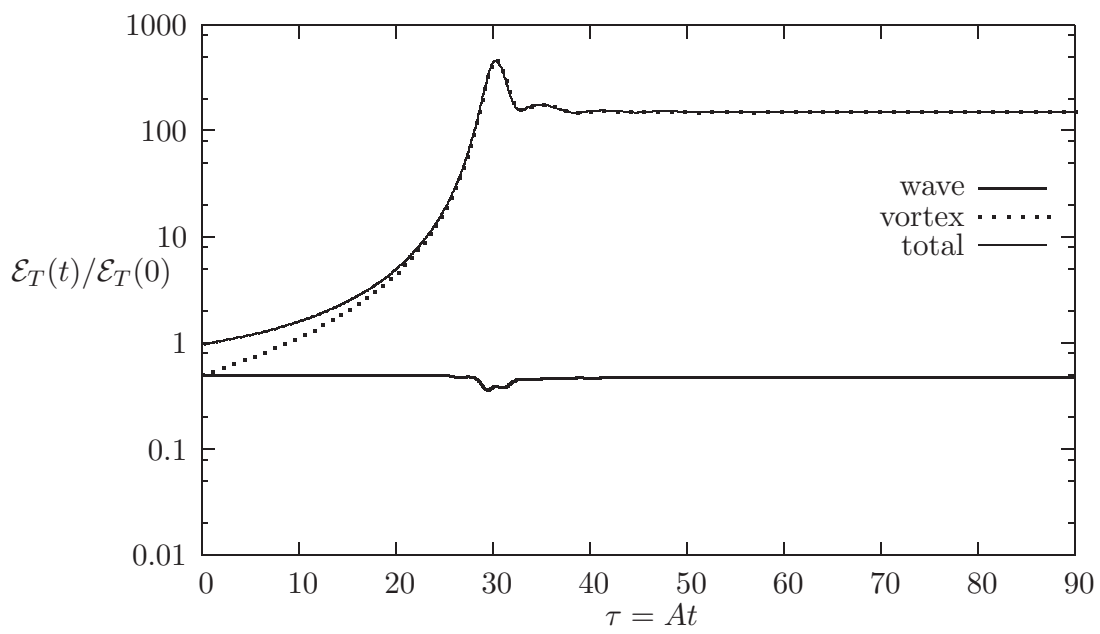

Figure 3. Time evolution of the transient growth rate $G(t)=\mathcal{E}_{T}(t) / \mathcal{E}_{T}(0)$ in vertically stable Keplerian disks $\left(R_{\Omega}=-4 / 3, R_{i}=1\right)$. The figure reveals that when $K_{2} / k_{1}=30(\gg 1)$ and $k_{3} / k_{1}=1$ (or $\sim 1$ ), there is an important transient growth rate that is mainly due to the vortex contribution (see also Figure 4).

i.e., it grows with time for $0 \leqslant \tau<\tau_{m} \equiv K_{2} / k_{1}$ and decays with time for $\tau>\tau_{m}$, approaching zero for long times. On the other hand, the wave energy remains constant, $\mathcal{E}_{T}^{(w)}\left(k_{3}=0, t\right) / \mathcal{E}_{T}(0)=1 / 2$, and hence, the total energy behaves as

$$
\frac{\mathcal{E}_{T}\left(k_{3}=0, \tau\right)}{\mathcal{E}_{T}(0)}=\frac{\mathcal{E}_{T}^{(w)}\left(k_{3}=0, \tau\right)}{\mathcal{E}_{T}(0)}+\frac{\mathcal{E}_{T}\left(k_{3}=0, \tau\right)}{\mathcal{E}_{T}(0)}=\frac{1}{2}+\frac{1}{2} \frac{K_{h}^{2}}{k_{h}^{2}}
$$

The maximum growth rate occurs at $\tau=\tau_{m}$ (or equivalently, for $k_{2}(t)=0$ ),

$$
\frac{\mathcal{E}_{T}\left(k_{3}=0, \tau_{m}\right)}{\mathcal{E}_{T}(0)}=1+\frac{1}{2} \frac{K_{2}^{2}}{k_{1}^{2}},
$$

and hence, at $K_{2} / k_{1} \gg 1$, it becomes very large. Note that the possible maximum value of $0<K_{2} / k_{1}$ is determined by the Reynolds number, which can take large values $\left(>10^{10}\right)$ in Keplerian disks (see, e.g., Tevzadze et al. 2003).

Recall that the results presented in this section concern Keplerian disks (i.e., $R_{\Omega}=-4 / 3$ ). Computations indicate that when $K_{2} / k_{1} \gg 1$ and $0 \leqslant k_{3}<k_{1}$ or $k_{3} \sim k_{1}$, the time evolution of the growth rate $\mathcal{E}_{T}(t) / \mathcal{E}_{T}(0)$ during the leading phase (i.e., $\left.\tau \leqslant \tau_{m}\right)$ follows Equation (39) in characterizing the dynamics in the $k_{3}=0$ plane (see Figure 3). The rapid transient growth is due to the vortex mode of the toroidal component since at $k_{3}=0$ it is found that all components $g_{i j}^{(v)}$ vanish except $g_{11}^{(v)}=K_{h} / k_{h}$, or equivalently (see Appendix A.4),

$$
u_{v}^{(1)}\left(k_{3}=0, \tau\right)=g_{1 j}^{(v)}\left(k_{3}=0, \tau\right) u_{0}^{(j)}=\frac{K_{h}}{k_{h}} u_{0}^{(1)} .
$$

A close examination of the "vortex" energy expression given by relation (36) indicates that the important contribution comes from terms (II) and (III) as shown by Figure 4. In fact, during the leading phase, term (II), which represents the "vortex" energy at $k_{3}=0$, is dominant. Whereas for $\tau>\tau_{m}$, term (III), which is associated with the vortex modes of the poloidal and buoyancy components $u_{v}^{(2)}$ and $u_{v}^{(3)}$, is dominant.

When $k_{3} / k_{1} \gg 1$, the transient growth is less important than in the case where $k_{3} \sim k_{1}$ even if $K_{2} / k_{1} \gg 1$ as shown by Figure 5 . This is mainly due to term (I) in Equation (36), which has a negative sign. Its magnitude, however, is large for $k_{3} / k_{1} \gg 1$.

To better characterize the contribution coming from the vortex mode to the total energy at different streamwise or vertical scales, we compute the power spectrum of the total energy (kinetic + potential) in the azimuthal or vertical directions. The power spectrum can be defined as

$$
S_{T}\left(k_{1}, t\right)=\int_{0}^{\infty} \int_{0}^{2 \pi} \mathcal{E}_{T}(t, \alpha, r) r d \alpha d r=\int_{0}^{\frac{\pi}{2}} \int_{0}^{2 \pi} \mathcal{E}_{T}(t, \alpha, \beta) \tan \beta\left(1+\tan ^{2} \beta\right) d \alpha d \beta,
$$

where $(r, \alpha)$ are polar coordinates in the $\left(K_{2}, k_{3}\right)$-plane,

$$
K_{2}=r \cos \alpha \quad k_{3}=r \sin \alpha, \text { and } \quad r=\tan \beta .
$$

The power spectrum of the total energy in the vertical direction, $S_{T}\left(k_{3}\right)$, can be defined similarly. As in several experimental and numerical studies, we use the following initial Gaussian spectrum (see, e.g., Hawley et al. 1996):

$$
\mathcal{E}_{T}(0)=\frac{C_{0}}{4 \pi k_{p}^{3}} \exp \left(-K^{2} / k_{p}^{2}\right)
$$




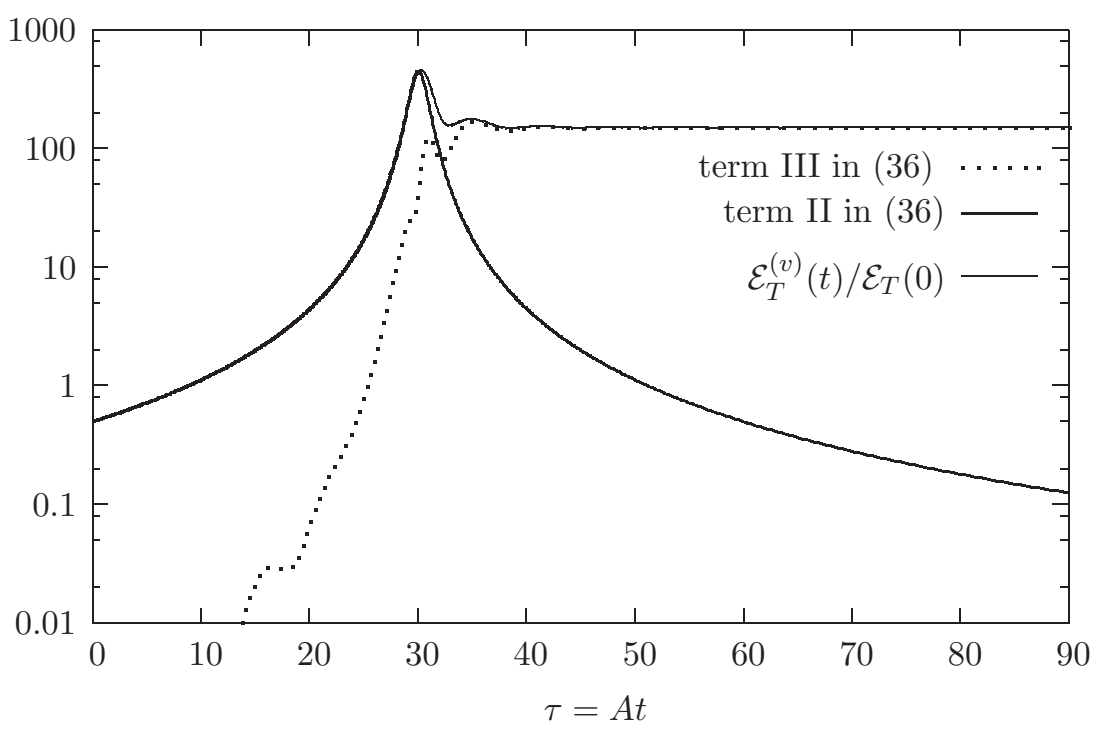

Figure 4. Relative contribution of terms (II) $\left(\sim K_{h}^{2} / k_{h}^{2}\right)$ and (III) in Equation (36) to the energy due to the vortex mode. This mode is an important contributor to the transient growth in vertically stratified Keplerian disks $\left(R_{i}=1\right)$. Here, $K_{2} / k_{1}=30$ and $k_{3} / k_{1}=1$, so the contribution coming from term (I) in Equation (36) is not important.

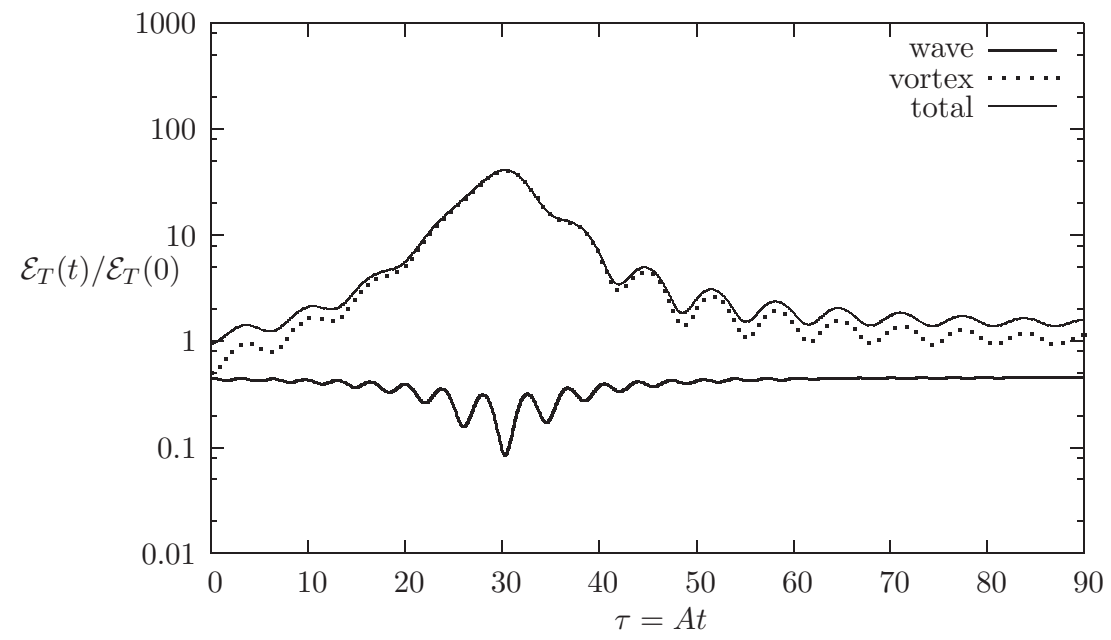

Figure 5. Time evolution of the transient growth rate $G(t)=\mathcal{E}_{T}(t) / \mathcal{E}_{T}(0)$ in vertically stable Keplerian disks $\left(R_{\Omega}=-4 / 3, R_{i}=1\right)$. The figure reveals that when $k_{3} / k_{1}(=10)$ is large, the transient growth is reduced even if $K_{2} / k_{1}(=30)$ is large.

where $C_{0}=4 E_{T}(0) / \sqrt{\pi}$ is the normalization constant and

$$
E_{T}(0)=4 \pi \int_{0}^{\infty} \mathcal{E}_{T}(0) K^{2} d K
$$

is the initial total energy that is equal to the initial kinetic energy since we have assumed that the initial potential energy is zero (see Section 4). We take $k_{p}=10$ in accordance with the experimental study by Piccirillo \& Van Atta (1997).

As indicated previously, when $k_{1} \neq 0$ and $k_{3} \neq 0$, the spectral density of energies given by Equations (35) and (36) is computed numerically. Also, the double integral described by Equation (40) is computed numerically using the Gauss-Chebyshev method with 400 points in the interval $0 \leqslant \beta \leqslant(\pi / 2)$ and 997 points in the interval $0 \leqslant \alpha \leqslant 2 \pi$.

Figure 6 shows the power spectrum $S_{T}\left(k_{1}\right)$ and the two parts $S_{T}^{(w)}\left(k_{1}\right)$ and $S_{T}^{(v)}\left(k_{1}\right)$ characterizing the contributions coming from the wave and the vortex modes for vertically stratified Keplerian disks $\left(R_{i}=1, R_{\Omega}=-4 / 3\right)$ at large times $(\tau=S t=40)$. The spectrum decreases with $k_{1}$, showing that there is more energy at large scales (i.e., low $k_{1}$ ). At these scales, there are small differences between $S_{T}\left(k_{1}\right)$ and $S_{T}^{(v)}\left(k_{1}\right)$, signifying that the contribution to the total energy from the vortex mode is dominant. The same conclusion is also drawn when considering the power spectrum of the total energy in the vertical direction, $S_{T}^{(v)}\left(k_{3}\right)$.

In addition, the present numerical results show that, at large times, only very low $k_{1}$ components continue to gain energy while at the other scales $S_{T}\left(k_{1}\right)$ is "time-independent" as illustrated by Figure 7. At $10^{-0.5}<k_{1}<10^{0.5}$, the power spectrum $S_{T}\left(k_{1}\right)$ scales as $k_{1}^{-a}$ where $a \approx 1$. This particular form is also found when considering the following initial spectrum with a $K^{2}$ form prefactor:

$$
\mathcal{E}_{T}(0)=\frac{C_{0}^{\prime}}{4 \pi k_{p}^{5}} K^{2} \exp \left(-K^{2} / k_{p}^{2}\right)
$$




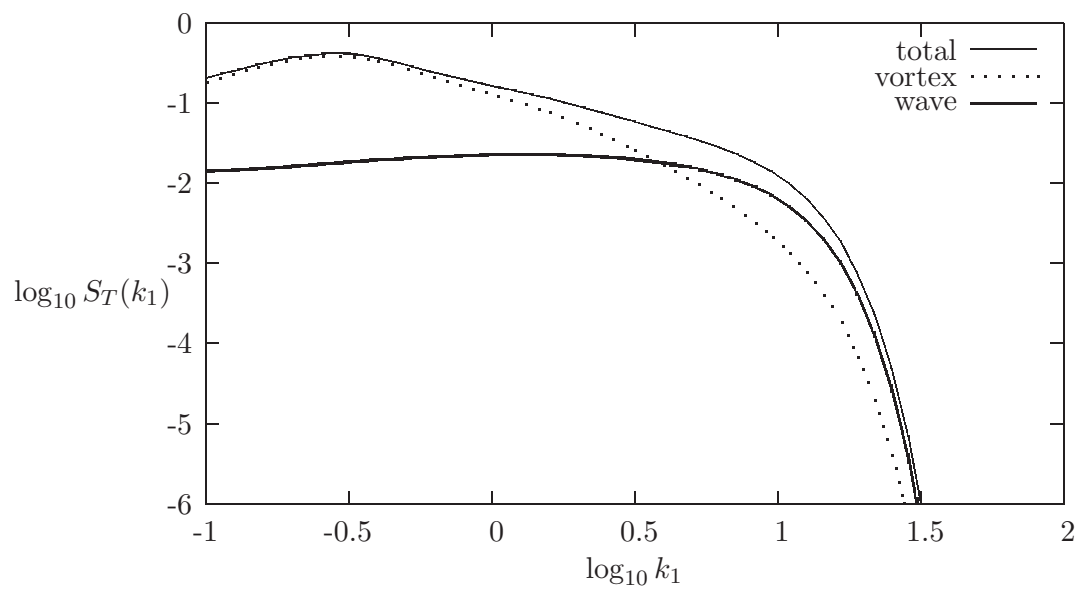

Figure 6. Power spectrum of total energy in the azimuthal direction for vertically stratified Keplerian disks with $R_{i}=1$ at $\tau=A t=40$. The figure clearly shows that the contribution coming from the vortex mode, $S_{T}^{(v)}\left(k_{1}\right)$, is dominant at large scales (i.e., at low $k_{1}$ ).

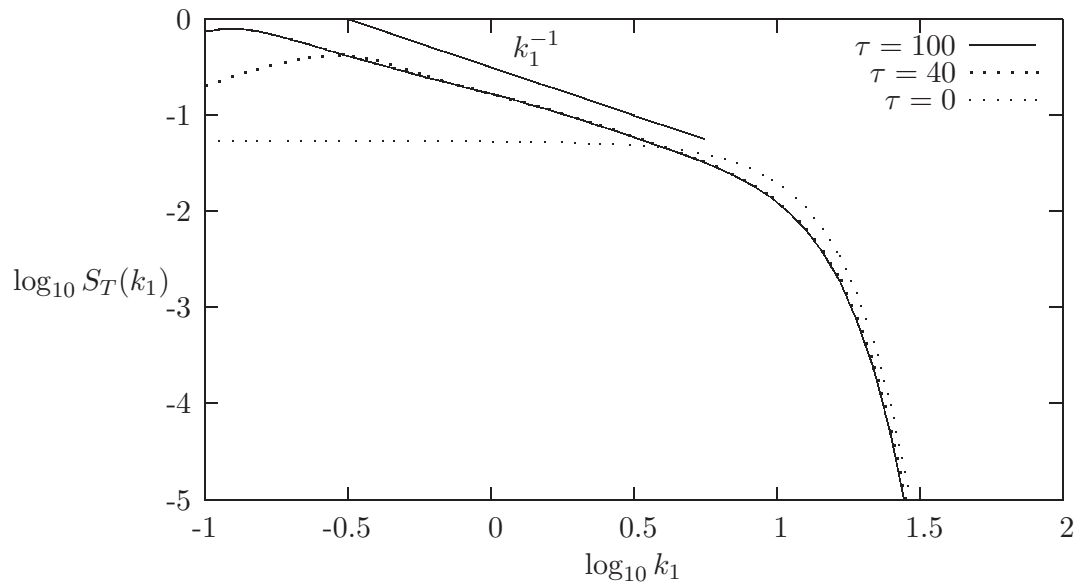

Figure 7. Power spectrum of total energy in the streamwise (azimuthal) direction $S_{T}\left(k_{1}\right)$ at different times for vertically stratified Keplerian disks with $R_{i}=1$. The figure shows that $S_{T}\left(k_{1}\right)$ scales as $k_{1}^{-a}$ where $a \approx 1$ in the range $0.3<k_{1}<3$. At this range, the power spectrum is "time-independent" for long times.

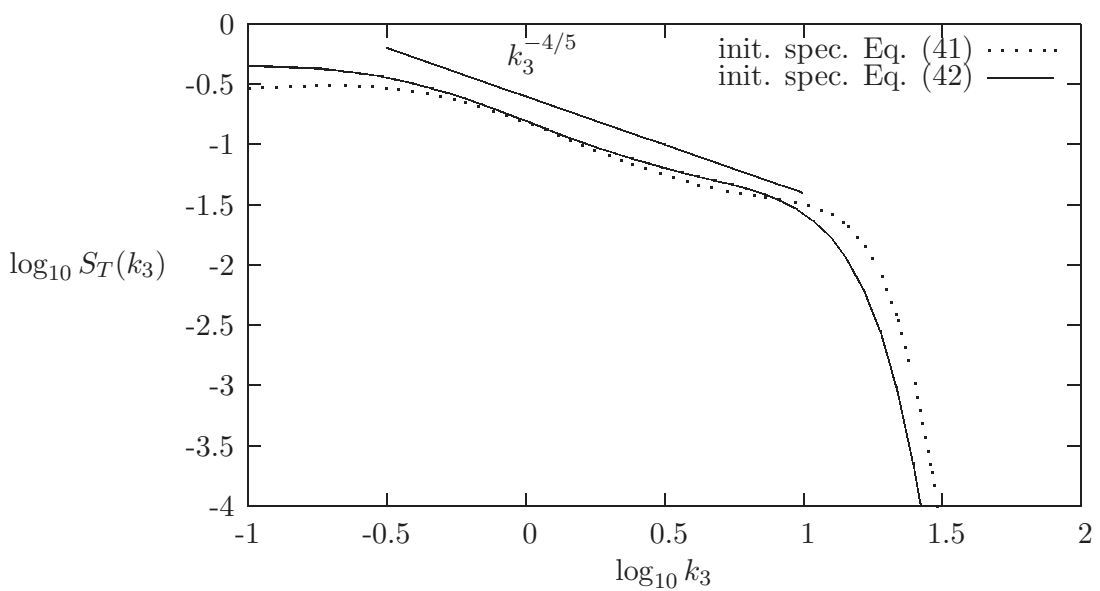

Figure 8. Power spectrum of the total energy in the vertical direction $S_{T}\left(k_{3}\right)$ at $A t=100$ for vertically stratified Keplerian disks with $R_{i}=1$. The figure shows that Figure 8. Power spectrum of the total energy in the vertical direction $S_{T}\left(k_{3}\right)$ at $A t=100$ for vertically stratified Keplerian disks with $R_{i}$
$S_{T}\left(k_{3}\right)$ scales as $k_{3}^{-a}$ for long times where $a \approx 4 / 5$ in the range $0.3<k_{1}<3$. At this range, the power spectrum is "time-independent."

where $C_{0}^{\prime}$ is the normalization constant. A similar behavior is found for the power spectrum of kinetic energy in the vertical direction, $S_{T}\left(k_{3}, t\right)$. At $10^{-0.5}<k_{1}<10^{0.5}, S_{T}\left(k_{3}\right)$ exhibits a $k^{-a}$ scaling where $a \approx 4 / 5$ for both spectra (41) and (42) as shown by Figure 8. Further discussion of the implications of these algebraic forms is given in Section 6. 


\section{DISKS WITH RADIAL AND VERTICAL STRATIFICATION}

\subsection{Stability Analysis of the Case with $k_{3}=0$}

When both radial and vertical stratification gradients are simultaneously present $\left(n_{2} \neq 0\right.$ and $\left.n_{3} \neq 0\right)$, the stability analysis under non-axisymmetric disturbances is much more complicated and requires tedious calculations except for the case with an infinite vertical wavelength $\left(k_{3}=0\right)$. The study of that case is relevant for the characterization of the rapid transient growth.

At $k_{3}=0$, such that $k_{h}=k$, the system (14) and the relation (16) reduce to

$$
\begin{gathered}
\frac{d g_{1 j}}{d \tau}=\frac{k_{1} k_{2}}{k_{h}^{2}} g_{1 j}+R_{i} n_{2} \frac{k_{1}}{k_{h}} g_{3 j}, \quad \frac{d g_{2 j}}{d \tau}=R_{i} n_{3} g_{3 j}, \\
\frac{d g_{3 j}}{d \tau}=-n_{2} \frac{k_{1}}{k_{h}} g_{1 j}-n_{3} g_{2 j}, \\
n_{3} g_{1 j}=n_{2} \frac{k_{1}}{k_{h}} g_{2 j}+\left[1-\delta\left(q_{0}\right)\right]\left[n_{3} \frac{K_{h}}{k_{h}} \delta_{1 j}-n_{2} \frac{k_{1}}{k_{h}} \delta_{2 j}\right],
\end{gathered}
$$

respectively. Thus, the substitution of Equation (44) into Equation (43) yields the following two-dimensional nonhomogeneous system:

$$
\frac{d}{d \tau}\left(\begin{array}{c}
g_{2 j} \\
n_{3} g_{3 j}
\end{array}\right)-\underbrace{\left(\begin{array}{cc}
0 & R_{i} \\
-n_{3}^{2}-n_{2}^{2} \frac{k_{1}^{2}}{k_{h}^{2}} & 0
\end{array}\right)}_{\mathbf{C}} \cdot\left(\begin{array}{c}
g_{2 j} \\
n_{3} g_{3 j}
\end{array}\right)=\left(\begin{array}{c}
0 \\
{\left[1-\delta\left(q_{0}\right)\right] h_{j}(\tau)}
\end{array}\right),
$$

where

$$
h_{j}(\tau)=-n_{2} \frac{k_{1}}{k_{h}}\left(n_{3} \frac{K_{h}}{k_{h}} \delta_{1 j}-n_{2} \frac{k_{1}}{k_{h}} \delta_{2 j}\right) .
$$

Using the same procedure as in Section 4, we show that the conditions (22) and (26) in Theorems 1 and 2 are satisfied by the system (45), signifying stability (see Appendix A.2).

We choose the following simple initial condition (see Appendix A.5) for the matrix $\mathbf{g}^{(w)}$, which is the solution of the system (45) at vanishing PV:

$$
g_{i j}^{(w)}(0)=\left(\begin{array}{ccc}
\frac{n_{2}}{n_{3}} \frac{k_{1}}{K_{h}} \beta_{0} & \frac{n_{2}}{n_{3}} \frac{k_{1}}{K_{h}} \beta_{1} & 0 \\
\beta_{0} & \beta_{1} & 0 \\
0 & 0 & \beta_{2}
\end{array}\right),
$$

and $g_{i j}^{(v)}(0)=\delta_{i j}-g_{i j}^{(w)}(0)$. As shown in Appendix A.5, this choice gives rise to vanishing mutual energies. Here,

$$
\begin{gathered}
-1 \leqslant \beta_{0}=\frac{\frac{n_{2}}{n_{3}} \frac{k_{1}}{K_{h}}}{1+\frac{n_{2}^{2}}{n_{3}^{2}} \frac{k_{1}^{2}}{K_{h}^{2}}} \leqslant 1, \\
0 \leqslant \beta_{1}=\frac{1}{1+\frac{n_{2}^{2}}{n_{3}^{2}} \frac{k_{1}^{2}}{K_{h}^{2}}} \leqslant 1, \quad \beta_{2}=1 .
\end{gathered}
$$

The expression of the spectral density of the wave energy is obtained by substituting Equation (44) with $\delta\left(q_{0}\right)=1$ into Equation (34):

$$
\frac{\mathcal{E}_{T}^{(w)}\left(k_{3}=0, t\right)}{\mathcal{E}_{T}(0)}=\frac{1}{2}\left[\left(1+\frac{n_{2}^{2}}{n_{3}^{2}} \frac{k_{1}^{2}}{k_{h}^{2}}\right)\left(\left|g_{21}^{(w)}\right|^{2}+\left|g_{22}^{(w)}\right|^{2}\right)+R_{i}\left(\left|g_{31}^{(w)}\right|^{2}+\left|g_{32}^{(w)}\right|^{2}\right)\right] .
$$

To compute the above expression, we integrate numerically the system (45) with $\delta\left(q_{0}\right)=0$ and the initial condition described by Equation (47). In a similar manner, we derive the spectral density of the vortex energy by substituting Equation (44) with $\delta\left(q_{0}\right)=0$ into Equation (34): 


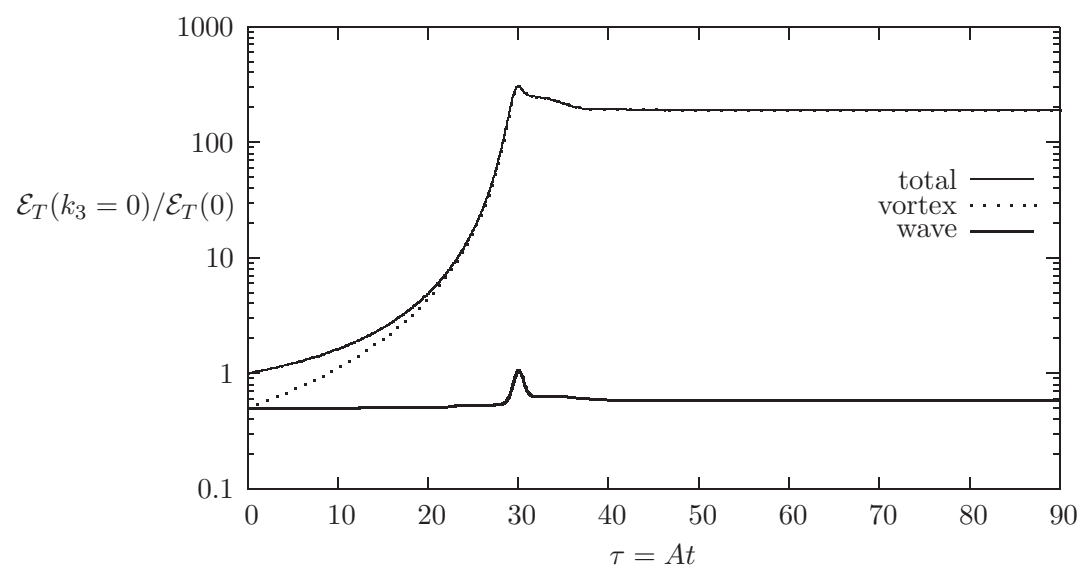

Figure 9. Time evolution of the transient growth rate $G(t)=\mathcal{E}_{T}(t) / \mathcal{E}_{T}(0)$ in Keplerian disks under combined vertical and radial stratification $\left(n_{2} / n_{3}=1, R_{i}=1\right)$. Here, $k_{3}=0$ and $K_{2} / k_{1}=30(\gg 1)$. During the leading phase $\tau<\tau_{m}=K_{2} / k_{1}$, the transient growth follows Equation (37) as in vertically stratified Keplerian disks.

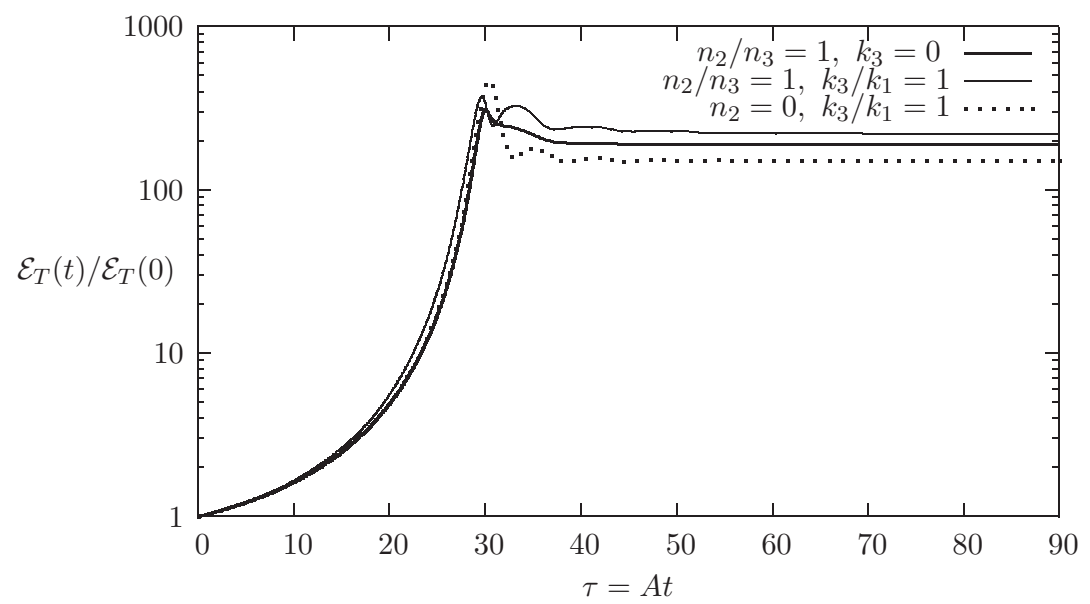

Figure 10. Transient growth rate $G(t)=\mathcal{E}_{T}(t) / \mathcal{E}_{T}(0)$ for $K_{2} / k_{1}=30$ and $k_{3} / k_{1}=1$ in vertically stratified Keplerian disks $\left(n_{2} / n_{3}=0\right)$ and Keplerian disks under combined radial and vertical stratification $\left(n_{2} / n_{3}=1\right)$. Here, $R_{i}=1$. The figure shows that the transient growth is approximately the same for stable vertical stratification and combined radial and vertical stratification.

$$
\begin{aligned}
& \frac{\mathcal{E}_{T}^{(v)}\left(k_{3}=0, t\right)}{\mathcal{E}_{T}(0)}=\underbrace{\frac{n_{2}}{n_{3}} \frac{k_{1}}{k_{h}^{2}} \Re g_{21}^{(v)}-\frac{n_{2}}{n_{3}} \frac{k_{1}^{2}}{k_{h}^{2}} \Re g_{22}^{(v)}}_{(I)}+\underbrace{\frac{1}{2}\left[\left(1+\frac{n_{2}^{2}}{n_{3}^{2}} \frac{k_{1}^{2}}{k_{h}^{2}}\right)\left|g_{22}^{(v)}\right|^{2}+R_{i}\left|g_{32}^{(v)}\right|^{2}\right]}_{(I I)}+ \\
& \underbrace{\frac{1}{2}\left(\frac{K_{h}^{2}}{k_{h}^{2}}+\frac{n_{2}^{2}}{n_{3}^{2}} \frac{k_{1}^{2}}{k_{h}^{2}}\right)}_{(I I I)}+\underbrace{\frac{1}{2}\left[\left(1+\frac{n_{2}^{2}}{n_{3}^{2}} \frac{k_{1}^{2}}{k_{h}^{2}}\right)\left|g_{21}^{(v)}\right|^{2}+R_{i}\left|g_{31}^{(v)}\right|^{2}\right]}_{(I V)} .
\end{aligned}
$$

We compute this expression by integrating numerically the system $(45)$ with $\delta\left(q_{0}\right)=0$ and the initial condition $g_{i j}^{(v)}(0)=\delta_{i j}-g_{i j}^{(w)}(0)$, where $g_{i j}^{(w)}(0)$ is given by Equation (47). When $k_{3} \neq 0$ and $k_{1} \neq 0$, we integrate numerically the system (14) with the initial condition $g_{i j}(0)=\delta_{i j}$ to compute $\mathcal{E}_{T}(t)$ given by Equation (34). As indicated previously, a fourth-order Runge-Kutta scheme with a time step of $\delta \tau=10^{-3}$ was used to perform the numerical integration.

\subsection{Results and Discussion}

As in the case of vertically stratified disks, numerical computation indicates that there is a rapid transient growth of $\mathcal{E}_{T}(t)$ provided $K_{2} / k_{1} \gg 1$ and $0 \leqslant k_{3} / k_{1}<1$ or $k_{3} \sim k_{1}$. Figure 9 shows the time evolution of the transient growth rate $G(t)=\mathcal{E}_{T}(t) / \mathcal{E}_{T}(0)$ and the contribution coming from the wave and vortex modes for stratified Keplerian disks with $R_{i}=1$ and $n_{2} / n_{3}=1$. Here, $K_{2} / k_{1}=30$ and $k_{3}=0$. As can be seen, the rapid transient growth is due to the vortex mode. During the phase $\tau<\tau_{m}=K_{2} / k_{1}$, the transient growth follows Equation (37), i.e.,

$$
\frac{\mathcal{E}_{T}(t)}{\mathcal{E}_{T}(0)}=\frac{1}{2}\left(1+\frac{K_{h}^{2}}{k_{h}^{2}}\right),
$$

as in vertically stratified Keplerian disks. Under the same conditions of Figure 9, except $k_{3} / k_{1}=1$, Figure 10 displays the time evolution of the growth rate $G(t)$. For comparison, we have reported in this figure the results at $k_{3}=0$ and also the results for 


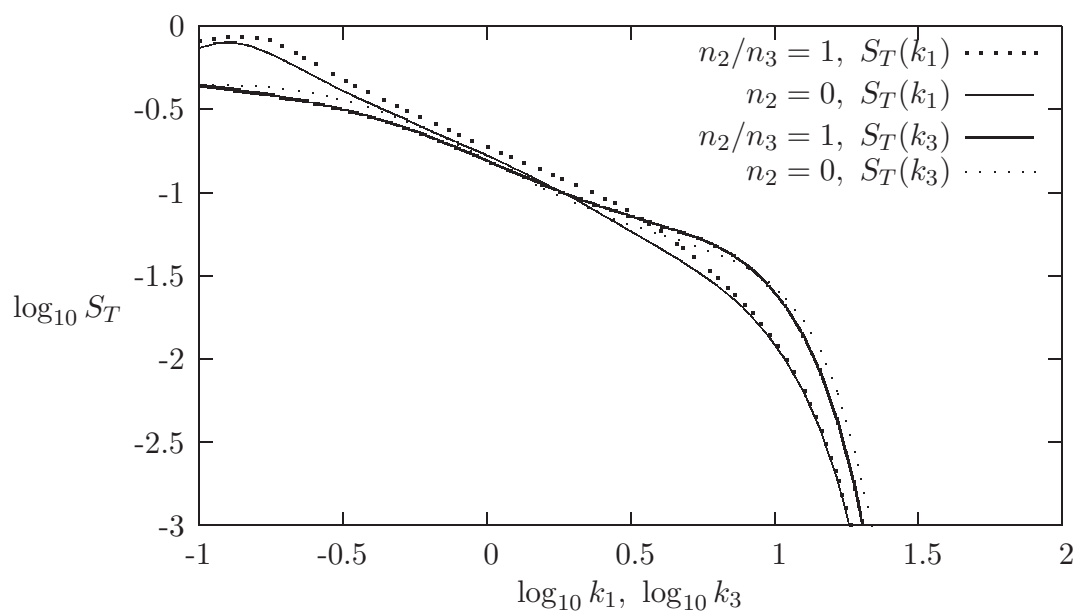

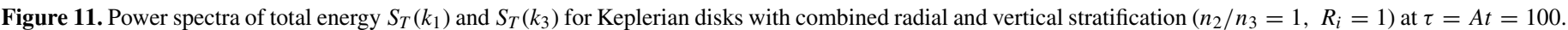
The figure shows that the shape of the spectra are similar to their counterparts in vertically stable, stratified Keplerian disks without radial stratification.

the vertically stratified disks $\left(n_{2} / n_{3}=0\right)$. At large times, the energy level is higher when radial and vertical buoyancy gradients are present than when vertical stratification alone is present. In both cases, the high level of energy is mainly due to the "vortex" component (in the sense of the particular solution of the nonhomogeneous reduced linear system of equations) of the poloidal and buoyancy modes, $u_{v}^{(2)}$ and $u_{v}^{(3)}$. More precisely, the high energy is due to the components $g_{21}^{(v)}$ and $g_{31}^{(v)}$, since

$$
\lim _{\tau \rightarrow \infty} \frac{\mathcal{E}_{T}(t)}{\mathcal{E}_{T}(0)}=\lim _{\tau \rightarrow \infty} \frac{\mathcal{E}_{T}^{(v)}(t)}{\mathcal{E}_{T}(0)}=\frac{1}{2}\left(\left|g_{21}^{(v)}\right|^{2}+R_{i}\left|g_{31}^{(v)}\right|^{2}\right)
$$

When both the radial and vertical buoyancy gradients are present, there is a similar behavior of the spectral density of the total energy considering either the $k_{3}=0$ mode or the modes for which $k_{1} \sim k_{3}$. This fully justifies the study of the $k_{3}=0$ mode to characterize the rapid transient growth that occurs when $K_{2} / k_{1} \gg 1$.

Finally, we present in Figure 11 the power spectra $S_{T}\left(k_{1}, \tau\right)$ and $S_{T}\left(k_{3}, \tau\right)$ in Keplerian disks with radial and vertical stratification $\left(n_{2} / n_{3}=1\right.$ and $\left.R_{i}=1\right)$ at the large time $\tau=A t=100$. For comparison, we have also reported in this figure $S_{T}\left(k_{1}, \tau\right)$ and $S_{T}\left(k_{3}, \tau\right)$, obtained in the case of a vertically stratified Keplerian disk $\left(n_{2} / n_{3}=0, R_{i}=1\right)$. As can be seen, there is little difference between the spectra in the two cases.

In previous studies, it was shown that the increase of the mean shear rate affects rather the large scales (low streamwise wavenumbers; see the experimental study by Nagata \& Komori 2000) in the sense that the turbulent structures become longer in the streamwise (azimuthal) direction, consistent with the generation of streaks. According to the study of homogeneous turbulent shear flow at a high mean shear rate, by means of SLT and direct numerical simulations (DNS; see Lee et al. 1990), the mechanism for the formation of streaks is mainly due to linear effects. In connection with the fact that the form $k_{1}^{-1}$, occurring at intermediate scales, would be an indicator of the presence of elongated structures in the streamwise (azimuthal) direction, it has been noted in the recent review of fluid mechanics in disks by Shariff (2009) that when the eddy Rossby number $R_{\omega} \equiv\left|\omega_{3}\right| /(2 \Omega)$ is very small, the resulting eddies are highly elongated in the azimuthal direction. Moreover, previous 2D incompressible (Bracco et al. 1999) and compressible (Goden \& Livio 2000; Johnson \& Gammie 2005b) disk simulations show the appearance of long-lived vortices that are anticyclonic; the weaker they are, the higher their aspect ratio becomes (see Section 5.6 in Shariff 2009).

\section{CONCLUDING REMARKS}

In this paper, we have shown several possible behaviors for Keplerian and sub-Keplerian disks with a Boussinesq fluid. Gravity is directed radially inward (see Figure 1(b)), while the radial density gradient is outward (linear stable case) and the vertical density gradient is positive (linear stable case). Indeed, apart from non-linear effects not considered here, either there is a dominant linear instability (with an exponential growth of perturbations with time) or the flow is linearly stable but it can be finite amplitude stable after suffering transient growths (that eventually decay if a finite viscosity is taken into account). We presume the conditions that have been discussed in Sections 5 (for vertical stable stratification) and Section 6 (under combined both stable radial and stable vertical stratifications), which are summarized below. The relevance of these behaviors to accretion disks is thought to be important even if the precise physical conditions acting on a real accretion disk remain for the moment still unknown. Let us recall first some former known situations: the stability criterion of Schwarzschild is that the flow is unstable if $N_{2}^{2}<0$, but with shear included we have the criterion of Solberg-Hoiland instead: the flow is unstable if $N_{2}^{2}+\kappa^{2}<0$. But there could be other situations where it can be Schwarzschild-unstable but Solberg-Hoiland-stable in the radial direction. Thus, we have examined here cases with $N_{2}^{2}>0$ and $N_{3}^{2}>0$ and we have first proved rigorous stability using the Levinson theorem, while also looking at possible transient growths.

As already explained in detail in our review in Section 1, our investigation is complementary to previous studies that have also examined stably stratified accretion-disk-like flows but in less general cases. More specifically, we have studied here the axisymmetric and non-axisymmetric linear theory for plane-wave disturbances within SLT of a rotating shear flow under combined radial and vertical stratification. We have applied the limit of a large speed of sound. We have considered the direct application of linear theory to astrophysical Keplerian accretion disks. 
Our main findings are summarized as follows.

1. We have derived a local dispersion equation for axisymmetric perturbations (see Equation (17)) showing that the astrophysical disks under combined stable radial and vertical stratification are linearly stable. The disks under unstable radial stratification $\left(N_{2}^{2}<0\right)$ are also linearly stable provided that the epicyclic frequency exceeds the critical buoyancy frequency, $\kappa_{N}$,

$$
\kappa^{2}>\kappa_{N}^{2}=\frac{N_{3}^{2}}{\left|n_{3}\right|} \frac{\left(n_{3} \cos \alpha-n_{2} \sin \alpha\right)^{2}}{\sin ^{2} \alpha} .
$$

Because of the relation $N_{3}^{2} / n_{2}=N_{2}^{2} / n_{3}$, the Solberg-Hoiland criterion (see, e.g., Lesur \& Papaloizou 2010) is recovered in the limit of $N_{3}=0$ (without vertical stratification); see above.

2. For neutral astrophysical accretion disks, the transient growth can act as an alternative to the more conventional MRI instability in ionized disks in generating the turbulence necessary to explain the observed accretion efficiencies (see, e.g., Afshordi et al. 2005). We have studied the transient energy growth of non-axisymmetric disturbances of disks under combined stable radial and vertical stratification. For the characterization of the transient energy growth, we have followed and extended previous works by Tevzadze et al. who studied the wave-vortex mode coupling in stable stratified Keplerian disks. These two modes are jointly able to extract the background energy and determine the disk dynamical activity in the small-scale range (see Tevzadze et al. 2008). Because the dynamics depends on which mode is initially imposed, we have introduced for each mode an associated Green's function with initial conditions leading to positive or zero values for the wave, vortex, and mutual energies. An initial balance of energy (i.e., the wave energy equal to the vortex energy, while the mutual energy is zero) was considered and the transient energy growth was computed for a Keplerian disk under combined stable radial and vertical stratification. A large transient energy growth was found for large radial wavenumbers $\left(K_{2} / k_{1} \gg 1, k_{3}=0\right.$, or $\left.k_{3} \sim k_{1}\right)$, as for stable vertically stratified astrophysical disks. The important transient energy growth is mainly due to the vortex mode. The contribution of the vortex mode to the power spectrum of energy in the azimuthal or vertical directions is important at large scales, while the contribution coming from the wave mode is important at small scales. At intermediate scales, the power spectrum is approximately time-independent for large times. The present study may stimulate disk simulations in the high Reynolds number regime. In addition, a detailed comparison between DNS and SLT would clarify the role of the linear processes in the turbulent angular momentum transport in disks and the corresponding flow structures. In addition, one should notice that the inclusion of stratification in numerical simulations involving the SB approximation is rather recent; see, for example, the paper by Meheut et al. (2010) for the role of vertical stratification on the Rossby wave instability in accretion disks. Another example is the influence of vertical stratification on the helicity conservation in the case of magneto-rotational turbulence by Oishi \& Mac Low (2011). Hence, we ascribe importance to disk stratification (vertical here) on the accretion dynamics.

\section{APPENDIX}

\section{A.1. Derivation of the Non-homogeneous System given by Equation (20)}

When there is no radial stratification, $n_{2}=0$, the differential system (14) reduces to

$$
\begin{gathered}
\frac{d g_{1 j}}{d \tau}=\frac{k_{1} k_{2}}{k_{h}^{2}} g_{1 j}-\left(1+R_{\Omega}\right) \frac{k_{3}}{k} g_{2 j}, \\
\frac{d g_{2 j}}{d \tau}=\left(R_{\Omega}+2 \frac{k_{1}^{2}}{k_{h}^{2}}\right) \frac{k_{3}}{k} g_{1 j}-\frac{k_{1} k_{2}}{k_{h}^{2}} \frac{k_{3}^{2}}{k^{2}} g_{2 j}+R_{i} \frac{k_{h}}{k} g_{3 j}, \\
\frac{d g_{3 j}}{d \tau}=-\frac{k_{h}}{k} g_{2 j},
\end{gathered}
$$

( $j=1,2,3)$. Due to the following relations:

$$
k \frac{d k}{d \tau}=k_{h} \frac{d k_{h}}{d \tau}=-k_{1} k_{2}, \quad \frac{d}{d \tau}\left(\frac{k}{k_{h}}\right)=\frac{k_{1} k_{2}}{k_{h}^{2}} \frac{k_{3}^{2}}{k_{h} k},
$$

the differential equation for $g_{2 j}$ can be rewritten as

$$
\frac{d}{d \tau}\left(\frac{k}{k_{h}} g_{2 j}\right)=\left(R_{\Omega}+2 \frac{k_{1}^{2}}{k_{h}^{2}}\right) \frac{k_{3}}{k_{h}} g_{1 j}+R_{i} g_{3 j},
$$

and the substitution of Equation (19) into the latter differential equation yields

$$
\frac{d}{d \tau}\left(\frac{k}{k_{h}} g_{2 j}\right)=C_{12}(\tau) g_{3 j}+h_{j}(\tau),
$$

where the expressions $C_{12}(\tau)$ and $h_{j}(\tau)$ are given by Equation (21). 


\section{A.2. Absolute Convergence of Some Integrals Appearing in Sections 4 and 6.}

The matrix $\mathbf{C}$ in the system (20) was decomposed into two matrices as $\mathbf{C}=\mathbf{C}_{0}+\mathbf{C}_{1}(\tau)$, where $\mathbf{C}_{\mathbf{0}}$ is a constant matrix. The nonzero components of the matrix $\mathbf{C}_{1}(\tau)$ in Equation (24) are

$$
\left(\mathbf{C}_{1}\right)_{12}=\left(1+R_{\Omega}\right)\left(R_{\Omega}+2 \frac{k_{1}^{2}}{k_{h}^{2}}\right) \frac{k_{3}^{2}}{k_{h}^{2}}, \quad\left(\mathbf{C}_{1}\right)_{21}=k_{3}^{2} / k^{2} .
$$

Then,

$$
\left|\left(\mathbf{C}_{1}\right)_{12}\right| \leqslant\left|R_{\Omega}\left(R_{\Omega}+1\right)\right| \frac{k_{3}^{2}}{k_{h}^{2}}+2\left|R_{\Omega}+1\right| \frac{k_{1}^{2} k_{3}^{2}}{k_{h}^{4}}
$$

and hence (see, e.g., Gradshteyn \& Ryzhik 1965),

$$
\begin{gathered}
\int_{0}^{\infty} \frac{k_{3}^{2}}{k_{h}^{2}} d \tau=\frac{k_{3}^{2}}{2 k_{1}^{2}}(\pi+2 \zeta)<\infty, \\
\int_{0}^{\infty} \frac{k_{3}^{2} k_{1}^{2}}{k_{h}^{4}} d \tau=\frac{k_{3}^{2}}{4 k_{1}^{2}}(\sin 2 \zeta+\pi+2 \zeta)<\infty,
\end{gathered}
$$

provided that $k_{1} \neq 0$. Here, $\tan \zeta=K_{2} / k_{1}$. This implies that

$$
\int_{0}^{\infty}\left|\left(\mathbf{C}_{\mathbf{1}}\right)_{12}(\tau)\right| d \tau<\infty
$$

In addition, one has

$$
\begin{gathered}
\int_{0}^{\infty}\left|\left(\mathbf{C}_{\mathbf{1}}\right)_{21}(\tau)\right| d \tau=\int_{0}^{\infty} \frac{k_{3}^{2}}{k^{2}} d \tau \\
=\frac{k_{3}^{2}}{k_{1} \sqrt{k_{1}^{2}+k_{3}^{2}}}\left[\frac{\pi}{2}+\arctan \left(\frac{K_{2}}{\sqrt{k_{1}^{2}+k_{3}^{2}}}\right)\right]<\infty
\end{gathered}
$$

if $k_{1}>0$ or

$$
=\frac{k_{3}^{2}}{\left|k_{1}\right| \sqrt{k_{1}^{2}+k_{3}^{2}}}\left[\frac{\pi}{2}-\arctan \left(\frac{K_{2}}{\sqrt{k_{1}^{2}+k_{3}^{2}}}\right)\right]<\infty
$$

if $k_{1}<0$ signifying that

$$
\int_{0}^{\infty}\left|\left(\mathbf{C}_{1}\right)_{21}(\tau)\right| d \tau<\infty
$$

The nonzero components of the vector $\boldsymbol{h}$ in the system (20) are $h_{1}(\tau)$ and $h_{3}(\tau)$, such that

$$
h_{1}(\tau)=k_{3} K_{h} h(\tau), \quad h_{3}(\tau)=k_{3}^{2} h(\tau), \text { and } \quad h(\tau)=\left(R_{\Omega}+2 \frac{k_{1}^{2}}{k_{h}^{2}}\right) \frac{1}{k_{h}^{2}} .
$$

Thus, it is sufficient to show that the latter term is impulsively small as $\tau \rightarrow \infty$ (see, e.g., Struble 1962)

$$
\begin{gathered}
\int_{0}^{\infty}|h(\tau)| d \tau=\int_{0}^{\infty}\left|\left(R_{\Omega}+2 \frac{k_{1}^{2}}{k_{h}^{2}}\right) \frac{1}{k_{h}^{2}}\right| \leqslant \\
\left|R_{\Omega}\right| \int_{0}^{\infty} \frac{1}{k_{h}^{2}} d \tau+2 \int_{0}^{\infty} \frac{k_{1}^{2}}{k_{h}^{4}} d \tau<\infty,
\end{gathered}
$$

provided that $k_{1} \neq 0$. 


\section{A.3. A Formal Analysis}

We consider the 2D homogeneous system associated with Equation (20), which is also reported here for the sake of clarity

$$
\frac{d}{d \tau}\left(\frac{k}{k_{h}} g_{2 j}^{(w)}\right)=C_{12} g_{3 j}^{(w)}, \quad \frac{d}{d \tau} g_{3 j}^{(w)}=C_{21}\left(\frac{k}{k_{h}} g_{2 j}^{(w)}\right),
$$

with the initial condition (30), or $g_{i j}^{(w)}=\delta_{i j}(i=1,2, j=1,2,3)$, while the component $g_{1 j}^{(w)}$ is expressed as a function of $g_{3 j}^{(w)}$, as done in Equation (27).

Let $\Psi_{1}(\tau)$ and $\Psi_{2}(\tau)$ be two linearly independent solutions of the above system. Then

$$
\begin{gathered}
g_{3 j}^{(w)}=A_{1 j} \Psi_{1}(\tau)+A_{2 j} \Psi_{2}(\tau), \quad(j=1,2,3), \\
g_{2 j}^{(w)}=-\frac{k}{k_{h}}\left(A_{1 j} \dot{\Psi}_{1}(\tau)+A_{2 j} \dot{\Psi}_{2}(\tau)\right),
\end{gathered}
$$

where $A_{1 j}$ and $A_{2 j}$ are constants depending on the initial conditions,

$$
\begin{gathered}
A_{1 j} \Psi_{1}(0)+A_{2 j} \Psi_{2}(0)=\delta_{3 j}, \\
A_{1 j} \dot{\Psi}_{1}(0)+A_{2 j} \dot{\Psi}_{2}(0)=-\frac{K_{h}}{K} \delta_{2 j},
\end{gathered}
$$

and $\dot{\Psi}_{1}=\left[d \Psi_{1}(\tau) / d \tau\right]$. Non-degenerate solutions are characterized by

$$
\Delta=\Psi_{1}(0) \dot{\Psi}_{2}(0)-\Psi_{2}(0) \dot{\Psi}_{1}(0) \neq 0 .
$$

This implies that $A_{11}=A_{21}=0$. Then,

$$
g_{31}^{(w)}=g_{21}^{(w)}=g_{11}^{(w)}=0
$$

at any time. Because $h_{2}(\tau)$ in the system (20) is zero and $g_{21}^{(v)}(0)=g_{22}^{(v)}(0)=0$, in a similar manner, we show that $g_{22}^{(v)}=g_{32}^{(v)}=0$ at any time.

\section{A.4. Analytical Solutions of the Modes $k_{1}=0$ and $k_{3}=0$}

For disks with vertical and radial stratification and $k_{1}=0$ (so that the wave vector and the matrix $\mathbf{L}$ are time-independent), an analytical solution of the system (12) is easily derived

$$
\begin{gathered}
u^{(2)}(t)=\underbrace{u_{0}^{(2)} \cos \left(\kappa_{s} t\right)+u_{0}^{(3)} \frac{\kappa_{s}}{A \cos \gamma} \sin \left(\kappa_{s} t\right.}_{\text {wave }}+\underbrace{\frac{q_{0}}{k} \frac{R_{\Omega} \sin \alpha}{\cos \gamma} \frac{A}{\kappa_{s}} \sin \left(\kappa_{s} t\right)}_{\text {vortex }}, \\
u^{(3)}(t)=-u_{0}^{(2)} \frac{A \cos \gamma}{\kappa_{s}} \sin \left(\kappa_{s} t\right)+u_{0}^{(3)} \cos \left(\kappa_{s} t\right)-\frac{q_{0}}{k} \frac{A^{2} R_{\Omega} \sin \alpha}{\kappa_{s}^{2}}\left(1-\cos \left(\kappa_{s} t\right)\right), \\
u^{(1)}(t)=-u_{0}^{(2)} \frac{A\left(1+R_{\Omega}\right) \sin \alpha}{\kappa_{s}} \sin \left(\kappa_{s} t\right)+u_{0}^{(3)} \frac{\left(1+R_{\Omega}\right) \sin \alpha}{\cos \gamma} \cos \left(\kappa_{s} t\right)+ \\
\frac{q_{0}}{k} \frac{1}{\cos \gamma}\left[1-\frac{A^{2} R_{\Omega}\left(1+R_{\Omega}\right) \sin ^{2} \alpha}{\kappa_{s}^{2}}\left(1-\cos \left(\kappa_{s} t\right)\right)\right],
\end{gathered}
$$

where $\kappa_{s}, \alpha$, and $\gamma$ are defined by Equation (17) and $q_{0}$ is described by Equation (19).

For vertically stratified disks $\left(n_{2}=0\right)$ and when $k_{3}=0$ so that $k=k_{h}$, an analytical solution for the system (12) is also easily derived,

$$
\begin{gathered}
\underbrace{u^{(1)}(t)}_{\text {vortex }}=\frac{q_{0}}{k_{h}}=u_{0}^{(1)} \frac{K_{h}}{k_{h}}, \\
\underbrace{u^{(2)}(t)}_{\text {wave }}=u_{0}^{(2)} \cos \left(N_{3} t\right)-u_{0}^{(3)} \sqrt{R_{i}} \sin \left(N_{3} t\right), \\
\underbrace{u^{(3)}(t)}_{\text {wave }}=u_{0}^{(2)} \frac{\sin \left(N_{3} t\right)}{\sqrt{R_{i}}}-u_{0}^{(3)} \cos \left(N_{3} t\right),
\end{gathered}
$$

indicating that the toroidal mode is a purely vortex mode. The poloidal and buoyancy modes constitute wave modes. 


\section{A.5. Isotropic Initial Conditions and the Realizability Conditions}

For clarity, we review here some previously given definitions and relationships.

We consider initial isotropic conditions for the second-order two-point correlation $\left\langle\boldsymbol{u}(\boldsymbol{x}, t) \boldsymbol{u}\left(\boldsymbol{x}^{\prime}, t\right)\right\rangle$ and a nonzero initial potential energy. We attempt to determine the initial conditions for the matrices $\mathbf{g}^{(w)}$ and $\mathbf{g}^{(v)}$ giving non-negative initial mutual energies.

Let $\rho$ be the density fluctuation and $\bar{\rho}$ the basic (or mean) density. The potential energy per unit mass is defined by

$$
E_{p}=-\frac{1}{2} \frac{g}{\rho_{0}\|\nabla \bar{\rho}\|}\left\langle\rho^{2}\right\rangle
$$

where the brackets \langle\rangle indicate an ensemble average and

$$
\|\nabla \bar{\rho}\|=\left[\left(\frac{\partial \bar{\rho}}{\partial x_{2}}\right)^{2}+\left(\frac{\partial \bar{\rho}}{\partial x_{3}}\right)^{2}\right]^{\frac{1}{2}}=\left[1+\frac{N_{2}^{4}}{N_{3}^{4}}\right]^{\frac{1}{2}}\left(-\frac{\rho_{0}}{g}\right) N_{3}^{2}=\left(-\frac{\rho_{0}}{g}\right) \frac{N_{3}^{2}}{n_{3}},
$$

since we have $n_{2} N_{3}^{2}=n_{3} N_{2}^{2}$, where $\boldsymbol{n}=\left(0, n_{2}, n_{3}\right)$ is a unit vector antiparallel to the gravitational acceleration $\mathbf{g}$ (see Salhi et al. 2012). Here, $N_{2}$ and $N_{3}$ are the Brunt-Väsäilä frequencies with respect to the $\left(x_{2}, x_{3}\right)$ directions,

$$
N_{2}^{2}=-\frac{g}{\rho_{0}}\left(\frac{\partial \bar{\rho}}{\partial x_{2}}\right), \quad N_{3}^{2}=-\frac{g}{\rho_{0}}\left(\frac{\partial \bar{\rho}}{\partial x_{3}}\right)
$$

in which $\rho_{0}$ is a reference density. Thus, the expression for $E_{p}$ becomes

$$
E_{p}=\frac{1}{2} \frac{g^{2} n_{3}}{\rho^{2} N_{3}^{2}}\left\langle\rho^{2}\right\rangle=\frac{1}{2} \frac{n_{3}}{N_{3}^{2}}\left\langle\theta^{2}\right\rangle=\frac{R_{i}}{2}\left\langle u_{p}^{2}\right\rangle,
$$

where

$$
\theta=\left(-\frac{g}{\rho_{0}}\right) \rho, \quad u_{p}=-\frac{n_{3} A}{N_{3}^{2}} \theta=\frac{n_{3} A}{\left(\partial \bar{\rho} / \partial x_{3}\right)} \rho,
$$

$R_{i}=N_{3}^{2} /\left(n_{3} A^{2}\right)$ is the Richardson number and $A$ is the shear rate, as already indicated.

Let $S_{\kappa}(K)$ and $S_{\rho}(K)$ be the radial spectra of the initial kinetic energy and potential energy, respectively,

$$
E_{\kappa}(0)=\int_{0}^{\infty} S_{\kappa}(K) d K, \quad E_{p}(0)=\int_{0}^{\infty} S_{\rho}(K) d K .
$$

As in previous studies of stratified homogeneous turbulence (see, e.g., Salhi \& Cambon 2007), we assume that the spectra $S_{\kappa}(K)$ and $S_{\rho}(K)$ are proportional

$$
S_{\rho}(K)=\xi S_{\kappa}(K),
$$

where $\xi$ is a positive coefficient. In addition, we consider initial isotropic conditions with zero initial helicity and zero value for the initial density fluxes,

$$
\begin{gathered}
\Re\left\langle\hat{u}_{i}(\boldsymbol{K}, 0) \hat{u}_{j}^{*}(\boldsymbol{K}, 0)\right\rangle=\frac{S_{\kappa}(K)}{4 \pi K^{2}}\left(\delta_{i j}-\frac{K_{i} K_{j}}{K^{2}}\right), \quad \Im\left\langle\hat{u}_{i}(\boldsymbol{K}, 0) \hat{u}_{j}^{*}(\boldsymbol{K}, 0)\right\rangle=0 \\
\left\langle\hat{u}_{p}(\boldsymbol{K}, 0) \hat{u}_{j}^{*}(\boldsymbol{K}, 0)\right\rangle=0 \quad(j=1,2,3),
\end{gathered}
$$

where the asterisk denotes conjugate and

$$
u_{p}(\boldsymbol{x}, t)=\hat{u}_{p}(\boldsymbol{k}, t) \exp (\imath \boldsymbol{k}(t) \cdot \boldsymbol{x}), \quad \hat{u}_{p}=u^{(3)} .
$$

In the orthonormal basis $\left(\boldsymbol{e}^{(1)}, \boldsymbol{e}^{(2)}, \boldsymbol{e}^{(3)}\right)$ where $\boldsymbol{e}^{(3)}=\boldsymbol{k} / k$, these initial conditions can be rewritten as

$$
\Re\left\langle u^{(i)}(\boldsymbol{K}, 0) u^{(j) *}(\boldsymbol{K}, 0)\right\rangle=\frac{S_{\kappa}(K)}{4 \pi K^{2}}\left(\begin{array}{ccc}
1 & 0 & 0 \\
0 & 1 & 0 \\
0 & 0 & \frac{2 \xi}{R_{i}}
\end{array}\right) \quad(i, j=1,2,3) .
$$


By setting $u^{(i)}(\boldsymbol{k}, t)=g_{i j}(t) u^{(j)}(\boldsymbol{K}, 0)$, the expression of the second-order spectral correlation can be expressed in terms of the Green's function $\mathrm{g}$ as follows:

$$
\Re\left\langle u^{(i)}(\boldsymbol{k}, t) u^{(j) *}(\boldsymbol{k}, t)\right\rangle=\frac{S_{\kappa}(K)}{4 \pi K^{2}} \Re\left[\left(\begin{array}{lll}
g_{11} & g_{12} & g_{13} \\
g_{21} & g_{22} & g_{23} \\
g_{31} & g_{32} & g_{33}
\end{array}\right) \cdot\left(\begin{array}{ccc}
g_{11}^{*} & g_{21}^{*} & g_{31}^{*} \\
g_{12}^{*} & g_{22}^{*} & g_{32}^{*} \\
\frac{2 \xi}{R_{i}} g_{13}^{*} & \frac{2 \xi}{R_{i}} g_{23}^{*} & \frac{2 \xi}{R_{i}} g_{33}^{*}
\end{array}\right)\right],
$$

where $g_{i j}(0)=\delta_{i j}$. Thus, we deduce expressions for the spectral density of toroidal, poloidal, and potential energies:

$$
\begin{gathered}
\mathcal{E}_{\text {tor }}(t)=\frac{1}{2}\left\langle u^{(1)}(\boldsymbol{k}, t) u^{(1) *}(\boldsymbol{k}, t)\right\rangle=\frac{S_{\kappa}(K)}{8 \pi K^{2}}\left(\left|g_{11}\right|^{2}+\left|g_{12}\right|^{2}+\frac{2 \xi}{R_{i}}\left|g_{13}\right|^{2}\right), \\
\mathcal{E}_{p o \ell}(t)=\frac{1}{2}\left\langle u^{(2)}(\boldsymbol{k}, t) u^{(2) *}(\boldsymbol{k}, t)\right\rangle=\frac{S_{\kappa}(K)}{8 \pi K^{2}}\left(\left|g_{21}\right|^{2}+\left|g_{22}\right|^{2}+\frac{2 \xi}{R_{i}}\left|g_{23}\right|^{2}\right), \\
\mathcal{E}_{\text {pot }}(t)=\frac{R_{i}}{2}\left\langle u^{(3)}(\boldsymbol{k}, t) u^{(3) *}(\boldsymbol{k}, t)\right\rangle=\frac{R_{i} S_{\kappa}(K)}{8 \pi K^{2}}\left(\left|g_{31}\right|^{2}+\left|g_{32}\right|^{2}+\frac{2 \xi}{R_{i}}\left|g_{33}\right|^{2}\right) .
\end{gathered}
$$

Hence, the spectral density of total (kinetic + potential) energy takes the form

$$
\mathcal{E}_{T}(t)=\frac{S_{K}(K)}{8 \pi K^{2}}\left(\sum_{i=1}^{2} \sum_{j=1}^{2}\left|g_{i j}\right|^{2}+R_{i} \sum_{j=1}^{2}\left|g_{3 j}\right|^{2}+2 \xi \sum_{i=1}^{3}\left|g_{i 3}\right|^{2}\right) .
$$

By setting $\xi=0$ in the above relation and $\mathcal{E}_{T}(0)=\mathcal{E}_{\kappa}(0)=S_{K}(K) /\left(4 \pi K^{2}\right)$, we recover Equation (34).

Additionally, we consider the relevant decomposition first proposed by Chagelishvili et al. (1997)

$$
u^{(i)}(\boldsymbol{k}, t)=\underbrace{u_{w}^{(i)}(\boldsymbol{k}, t)}_{\text {wave }}+\underbrace{u_{v}^{(i)}(\boldsymbol{k}, t)}_{\text {vortex }}=g_{i j}(t) u^{(j)}(\boldsymbol{K}, 0),
$$

so that

$$
\begin{gathered}
u_{w}^{(i)}(\boldsymbol{k}, t)=g_{i j}^{(w)}(t) u^{(j)}(\boldsymbol{K}, 0), \quad u_{v}^{(i)}(\boldsymbol{k}, t)=g_{i j}^{(v)}(t) u^{(j)}(\boldsymbol{K}, 0), \\
g_{i j}(t)=g_{i j}^{(w)}(t)+g_{i j}^{(v)}(t)
\end{gathered}
$$

for any time, where $g_{i j}^{(w)}$ is the Green's function characterizing the "wave" and "vortex" regimes, respectively, i.e., the regimes corresponding to a zero value or a nonzero value for the PV. For instance, by substituting the relation (A10) into Equation (A7), we obtain the expressions for the spectral density of the toroidal wave, vortex and mutual energies,

$$
\begin{gathered}
\mathcal{E}_{\text {tor }}^{(w)}(t)=\frac{1}{2}\left\langle u_{w}^{(1)}(\boldsymbol{k}, t) u_{w}^{(1) *}(\boldsymbol{k}, t)\right\rangle=\frac{S_{\kappa}(K)}{8 \pi K^{2}}\left(\left|g_{11}^{(w)}\right|^{2}+\left|g_{12}^{(w)}\right|^{2}+\frac{2 \xi}{R_{i}}\left|g_{13}^{(w)}\right|^{2}\right), \\
\mathcal{E}_{\text {tor }}^{(v)}(t)=\frac{1}{2}\left\langle u_{v}^{(1)}(\boldsymbol{k}, t) u_{v}^{(1) *}(\boldsymbol{k}, t)\right\rangle=\frac{S_{\kappa}(K)}{8 \pi K^{2}}\left(\left|g_{11}^{(v)}\right|^{2}+\left|g_{12}^{(v)}\right|^{2}+\frac{2 \xi}{R_{i}}\left|g_{13}^{(v)}\right|^{2}\right), \\
\mathcal{E}_{\text {tor }}^{(v w)}(t)=\Re\left\langle u_{w}^{(1)}(\boldsymbol{k}, t) u_{v}^{(1) *}(\boldsymbol{k}, t)\right\rangle=\frac{S_{\kappa}(K)}{4 \pi K^{2}} \Re\left(g_{11}^{(w)} g_{11}^{(v) *}+g_{12}^{(w)} g_{12}^{(v) *}+\frac{2 \xi}{R_{i}} g_{13}^{(w)} g_{13}^{(v) *}\right) .
\end{gathered}
$$

In the following, we will examine the initial condition $g_{i j}^{(w)}(0)$ giving a positive or a zero value for the initial spectral density of the mutual energies. In fact, to ensure the realizability conditions, it is necessary that the kinetic energy be positive or zero. Also, the potential energy must be positive or zero since $R_{i}>0$ (stable stratification). We assume that $g_{i j}^{(w)}(0)$ is real. Because $g_{i j}(0)=\delta_{i j}$, and hence, $g_{i j}^{(v)}(0)=\delta_{i j}-g_{i j}^{(w)}(0)$ at $t=0$, Equation (A7) reduces to

$$
\mathcal{E}_{\text {tor }}^{(v w)}(0)=\frac{S_{\kappa}(K)}{4 \pi K^{2}}\left[g_{11}^{(w)}(0)-\left(\left|g_{11}^{(w)}(0)\right|^{2}+\left|g_{12}^{(w)}(0)\right|^{2}+\frac{2 \xi}{R_{i}}\left|g_{13}^{(w)}(0)\right|^{2}\right)\right] .
$$


Similarly, we obtain

$$
\begin{aligned}
& \mathcal{E}_{p o \ell}^{(v w)}(0)=\frac{S_{\kappa}(K)}{4 \pi K^{2}}\left[g_{22}^{(w)}(0)-\left(\left|g_{21}^{(w)}(0)\right|^{2}+\left|g_{22}^{(w)}(0)\right|^{2}+\frac{2 \xi}{R_{i}}\left|g_{23}^{(w)}(0)\right|^{2}\right)\right] \\
& \mathcal{E}_{\text {pot }}^{(v w)}(0)=R_{i} \frac{S_{\kappa}(K)}{4 \pi K^{2}}\left[\frac{2 \xi}{R_{i}} g_{33}^{(w)}(0)-\left(\left|g_{31}^{(w)}(0)\right|^{2}+\left|g_{32}^{(w)}(0)\right|^{2}+\frac{2 \xi}{R_{i}}\left|g_{33}^{(w)}(0)\right|^{2}\right)\right] .
\end{aligned}
$$

Because the mutual energy must be positive or zero, this implies that

$$
\begin{gathered}
g_{11}^{(w)}(0) \geqslant\left(\left|g_{11}^{(w)}(0)\right|^{2}+\left|g_{12}^{(w)}(0)\right|^{2}+\frac{2 \xi}{R_{i}}\left|g_{13}^{(w)}(0)\right|^{2}\right), \\
g_{22}^{(w)}(0) \geqslant\left(\left|g_{21}^{(w)}(0)\right|^{2}+\left|g_{22}^{(w)}(0)\right|^{2}+\frac{2 \xi}{R_{i}}\left|g_{23}^{(w)}(0)\right|^{2}\right), \\
g_{33}^{(w)}(0) \geqslant \frac{R_{i}}{2 \xi}\left(\left|g_{31}^{(w)}(0)\right|^{2}+\left|g_{32}^{(w)}(0)\right|^{2}+\frac{2 \xi}{R_{i}}\left|g_{33}^{(w)}(0)\right|^{2}\right) .
\end{gathered}
$$

\section{A.5.1. Vertically-Stratified Disks}

We will now consider the case of vertically stratified disks. In that case, the equation characterizing a vanishing PV can be written as

$$
g_{1 j}^{(w)}(t)=\left(1+R_{\Omega}\right) \frac{k_{3}}{K_{h}} g_{3 j}^{(w)}(t) \quad(j=1,2,3),
$$

setting $\delta\left(q_{0}\right)=0$ in Equation (19). Accordingly, Equation (A11) reduces to

$$
\left(1+R_{\Omega}\right) \frac{k_{3}}{K_{h}} g_{31}^{(w)}(0) \geqslant\left(1+R_{\Omega}\right)^{2} \frac{k_{3}^{2}}{K_{h}^{2}}\left(\left|g_{31}^{(w)}(0)\right|^{2}+\left|g_{32}^{(w)}(0)\right|^{2}+\frac{2 \xi}{R_{i}}\left|g_{33}^{(w)}(0)\right|^{2}\right) \text {. }
$$

For the sake of simplicity, we consider the following form:

$$
g_{i j}^{(w)}(0)=\left(\begin{array}{ccc}
0 & \chi_{0} & 0 \\
\chi_{1} & 0 & \chi_{2}
\end{array}\right) \quad(i=2,3, j=1,2,3),
$$

where $\chi_{0}, \chi_{1}$, and $\chi_{2}$ are arbitrary coefficients. The substitution of this form into Equations (A12)-(A14) yields

$$
\left\{\begin{array}{c}
\chi_{0} \geqslant \chi_{0}^{2}, \\
\left(1+R_{\Omega}\right) \frac{k_{3}}{K_{h}} \chi_{1} \geqslant\left(1+R_{\Omega}\right)^{2} \frac{k_{3}^{2}}{K_{h}^{2}}\left(\chi_{1}^{2}+\frac{2 \xi}{R_{i}} \chi_{2}^{2}\right) . \\
\frac{2 \xi}{R_{i}} \chi_{2} \geqslant\left(\chi_{1}^{2}+\frac{2 \xi}{R_{i}} \chi_{2}^{2}\right)
\end{array}\right.
$$

When $\xi=0$, as in the present study, the above inequalities lead to $\chi_{1}=0$ and $0 \leqslant \chi_{0} \leqslant 1$, while $\chi_{2}$ can take any positive or zero value. This justifies the choice of the initial condition described by Equation (30) that gives a zero value for the mutual energies. For the case where the initial potential energy is not zero, $\xi \neq 0$, the vanishing initial mutual energies correspond to

$$
\chi_{0}=1, \quad \chi_{1}=0, \quad \chi_{2}=1,
$$

when $k_{3}=0$ or $R_{\Omega}=-1$ (i.e., the case of zero absolute vorticity) and

$$
\chi_{0}=1, \quad\left(\chi_{1}^{2}+\frac{2 \xi}{R_{i}} \chi_{2}^{2}\right)=\frac{K_{h}}{\left(1+R_{\Omega}\right) k_{3}} \chi_{1}=\frac{2 \xi}{R_{i}} \chi_{2}
$$

or equivalently,

$$
\begin{gathered}
\chi_{0}=1, \quad-1 \leqslant \chi_{1}=\frac{\frac{2 \xi}{R_{i}}\left(1+R_{\Omega}\right) \frac{k_{3}}{K_{h}}}{\left(1+\frac{2 \xi}{R_{i}}\left(1+R_{\Omega}\right)^{2} \frac{k_{3}^{2}}{K_{h}^{2}}\right)} \leqslant 1 \\
0 \leqslant \chi_{2}=\frac{1}{\left(1+\frac{2 \xi}{R_{i}}\left(1+R_{\Omega}\right)^{2} \frac{k_{3}^{2}}{K_{h}^{2}}\right)} \leqslant 1
\end{gathered}
$$

otherwise. 


\section{A.5.2. Disks with Combined Radial and Vertical Stratification}

When there is combined radial and vertical stratification $\left(n_{2} / n_{3} \neq 0\right)$ and $k_{3}=0$ (an infinite vertical wavelength), the equation characterizing the conservation of the PV gives

$$
g_{1 j}^{(w)}(t)=\frac{n_{2}}{n_{3}} \frac{k_{1}}{k_{h}} g_{2 j}^{(w)}(t),
$$

setting $\delta\left(q_{0}\right)=0$ and $k_{3}=0$ in Equation (16). In that case, we consider the following form:

$$
g_{i j}^{(w)}(0)=\left(\begin{array}{ccc}
\beta_{0} & \beta_{1} & 0 \\
0 & 0 & \beta_{2}
\end{array}\right) \quad(i=2,3, j=1,2,3)
$$

and for vanishing mutual energies we obtain, by proceeding in a similar manner, the following expressions:

$$
\begin{aligned}
& -1 \leqslant \beta_{0}=\frac{\frac{n_{2}}{n_{3}} \frac{k_{1}}{K_{h}}}{1+\frac{n_{2}^{2}}{n_{3}^{2}} \frac{k_{1}^{2}}{K_{h}^{2}}} \leqslant 1, \\
& 0 \leqslant \beta_{1}=\frac{1}{1+\frac{n_{2}^{2}}{n_{3}^{2}} \frac{k_{1}^{2}}{K_{h}^{2}}} \leqslant 1, \quad \beta_{2}=1
\end{aligned}
$$

that are independent of the Richardson number $R_{i}$.

\section{REFERENCES}

Afshordi, N., Mukhopadhyay, B., \& Narayan, R. 2005, ApJ, 629, 373

Balbus, S. A. 2003, ARA\&A, 41, 555

Balbus, S. A. 2011, Natur, 470, 475

Balbus, S. A., \& Hawley, J. F. 1991, ApJ, 376, 214

Balbus, S. A., \& Hawley, J. F. 1992, ApJ, 400, 610

Balbus, S. A., \& Hawley, J. F. 1998, RvMP, 70, 1

Balbus, S. A., \& Hawley, J. F. 2006, ApJ, 652, 1020

Blackburn, H. M., Barkley, D., \& Sherwin, S. J. 2008, JFM, 603, 271

Boberg, L., \& Brosa, U. 1988, ZNatA, 43a, 697

Bodo, G., Chagelishvili, G., Murante, G., et al. 2005, A\&A, 437, 9

Brandenburg, A., \& Dintrans, B. 2006, A\&A, 450, 437

Bracco, A., Chavanis, P., Provenzale, A., \& Spiegel, E. 1999, PhFl, 11, 2280

Butler, K. M., \& Farrell, B. F. 1992, PhFl, 4, 1637

Chagelishvili, G. D., Tevzadze, A. G., Bodo, G., \& Moiseev, S. S. 1997, PhRvL, 79,3178

Chagelishvili, G. D., Zahn, J.-P., Tevzadze, A. G., \& Lominadze, J. G. 2003, A\&A, 402, 401

Chandrasekhar, S. 1961, Hydrodynamic and Hydromagnetic Stability (Oxford: Clarendon)

Craik, A. D. D. 1989, JFM, 198, 275

Drazin, P. G., \& Reid, W. H. 1981, Hydrodynamic Stability (Cambridge: Cambridge Univ. Press)

Dubrulle, B., Marié, L., Normand, Ch., et al. 2005, A\&A, 429, 1

Eastham, M. S. P. 1989, The Asymptotic Solution of Linear Differential Systems, Applications of the Levinson Theorem (Oxford: Clarendon)

Gammie, C. F., \& Menou, K. 1998, ApJ, 492, 75

Goden, P., \& Livio, M. 2000, MNRAS, 213, 7

Goldreich, P., \& Lynden-Bell, D. 1965, MNRAS, 130, 125

Gradshteyn, I. S., \& Ryzhik, I. M. 1965, Table of Integrals, Series, and Products (New York: Academic)

Grossmann, S. 2000, RvMP, 72, 603

Hawley, J. F., Gammie, C. F., \& Balbus, S. A. 1995, ApJ, 440, 712

Hawley, J. F., Gammie, C. F., \& Balbus, S. A. 1996, ApJ, 464, 690

Ioannou, P. J., \& Kakouris, A. 2001, ApJ, 550, 931

Ji, H., Burin, M., Schartman, E., \& Goodman, J. 2006, Natur, 444, 343

Johnson, B. M., \& Gammie, C. F. 2005a, ApJ, 626, 978

Johnson, B. M., \& Gammie, C. F. 2005b, ApJ, 635, 149

Klar, H. H. 2004, ApJ, 606, 1070

Klar, H. H., \& Bodenheimer, P. 2003, ApJ, 582, 869

Lagnado, R. R., Phan-Thien, N., \& Leal, L. G. 1984, PhFl, 27, 1094

Le Bars, M., \& Le Gal, P. 2007, PhRvL, 99, 064502
Lee, M. J., Kim, J., \& Moin, P. 1990, JFM, 216, 561

Lesur, G., \& Longaretti, P. 2007, MNRAS, 378, 1471

Lesur, G., \& Papaloizou, 2010, ApJ, 725, 146

Longaretti, P. 2002, ApJ, 576, 587

McWilliams, J. C., \& Yavneh, I. 1998, PhFl, 10, 2587

Meheut, H., Casse, F., Varniere, P., \& Tagger, M. 2010, A\&A, 516, 31

Menou, K. 2000, Sci, 288, 2022

Moffatt, H. K. 1967, in The Interaction of Turbulence with Strong Wind Shear, Colloquium on Atmospheric Turbulence and Radio Wave Propagation, ed. A. M. Yaglom \& V. I. Tatarsky (Nauka: Moscow), 139

Molemaker, M. J., McWilliams, J. C., \& Yavneh, I. 2001, PhRvL, 86, 5270

Mukhopadhyay, B., Afshordi, N., \& Narayan, R. 2005, ApJ, 629, 383

Nagata, K., \& Komori, S. 2000, JFM, 408, 39

Oishi, J. S., \& Mac Low, M. M. 2011, ApJ, 740, 18

Paoletti, M. S., van Gils, D. P. M., Dubrulle, B., et al. 2012, A\&A, 547, A64

Piccirillo, P. S., \& Van Atta, C. W. 1997, JFM, 334, 61

Reddy, S. C., \& Henningson, D. S. 1993, SJAM, 53, 15

Riedinger, X., le Dizès, S., \& Meunier, P. 2011, JFM, 672, 130

Regev, O., \& Umurhan, O. M. 2008, A\&A, 481, 21

Salhi, A., \& Cambon, C. 2010, PhRvE, 81, 026302

Salhi, A., \& Cambon, C. 2007, PhFl, 19, 055102

Salhi, A., \& Cambon, C. 1997, JFM, 347, 171

Salhi, A., Cambon, C., \& Speziale, C. G. 1997, PhFl, 9, 2300

Salhi, A., Lehner, T., Godeferd, F., \& Cambon, C. 2012, PhRvE, 85, 026301

Schmid, P. J., \& Henningson, D. S. 2001, Stability and Transition in Shear Flows (Berlin: Springer)

Shakura, N. I., \& Sunyaev, R. A. 1973, A\&A, 24, 337

Shariff, K. 2009, AnRFM, 41, 283

Schartman, E., Ji, H., Burin, M. J., \& Goodman, J. 2012, A\&A, 543, A94

Stone, J. M., Gammie, C. F., Balbus, S. A., \& Hawley, J. F. 2000, Protostars and Planets IV (Tucson, AZ: Univ. Arizona Press), 589

Struble, R. A. 1962, Nonlinear Differential Equations (New York: McGrawHill)

Tevzadze, A. G., Chagelishvili, G. D., Zahn, J.-P., Chanishvili, R. G., \& Lominadze, J. G. 2003, A\&A, 407, 779

Tevzadze, A. G., Chagelishvili, G. D., Bodo, G., \& Rossi, P. 2010, MNRAS, 401, 901

Tevzadze, A. G., Chagelishvili, G. D., \& Zahn, J.-P. 2008, A\&A, 478, 9

Umurhan, O. M. 2006, MNRAS, 365, 85

Umurhan, O. M. 2008, A\&A, 489, 953

Umurhan, O. M., \& Regev, O. 2004, A\&A, 427, 855

Yecko, P. A. 2004, A\&A, 425, 385 